\title{
REVISED Breeding novel solutions in the brain: A model of
}

\section{Darwinian neurodynamics [version 2; peer review: 3}

\section{approved]}

\author{
András Szilágyi ${ }^{1-3^{*}}$, István Zachar ${ }^{1,3,4^{*}}$, Anna Fedor ${ }^{1-3}$, Harold P. de Vladar ${ }^{1,3}$, \\ Eörs Szathmáry (iD) 1-5
}

\footnotetext{
${ }_{1}^{1}$ Parmenides Center for the Conceptual Foundations of Science, Munich/Pullach, 82049, Germany

${ }_{2}^{2}$ MTA-ELTE Theoretical Biology and Evolutionary Ecology Research Group, Budapest, H-1117, Hungary

${ }^{3}$ Institute of Advanced Studies, Kőszeg, H-9730, Hungary

${ }^{4}$ Department of Plant Systematics, Ecology and Theoretical Biology, Institute of Biology, Eötvös University, Budapest, H-1117, Hungary

${ }^{5}$ Evolutionary Systems Research Group, MTA Ecological Research Centre, Tihany, Hungary

* Equal contributors
}

V2 First published: 28 Sep 2016, 5:2416

https://doi.org/10.12688/f1000research.9630.1

Latest published: 29 Jun 2017, 5:2416

https://doi.org/10.12688/f1000research.9630.2

\section{Abstract}

Background: The fact that surplus connections and neurons are pruned during development is well established. We complement this selectionist picture by a proof-of-principle model of evolutionary search in the brain, that accounts for new variations in theory space. We present a model for Darwinian evolutionary search for candidate solutions in the brain.

Methods: We combine known components of the brain - recurrent neural networks (acting as attractors), the action selection loop and implicit working memory - to provide the appropriate Darwinian architecture. We employ a population of attractor networks with palimpsest memory. The action selection loop is employed with winners-share-all dynamics to select for candidate solutions that are transiently stored in implicit working memory.

Results: We document two processes: selection of stored solutions and evolutionary search for novel solutions. During the replication of candidate solutions attractor networks occasionally produce recombinant patterns, increasing variation on which selection can act. Combinatorial search acts on multiplying units (activity patterns) with hereditary variation and novel variants appear due to (i) noisy recall of patterns from the attractor networks, (ii) noise during transmission of candidate solutions as messages between networks, and,

(iii) spontaneously generated, untrained patterns in spurious

attractors.

Conclusions: Attractor dynamics of recurrent neural networks can be

\section{Open Peer Review \\ Approval Status \\ 12 \\ 23 \\ version 2 \\ (revision) \\ 29 Jun 2017 \\ version 1 \\ 28 Sep 2016

$\checkmark$
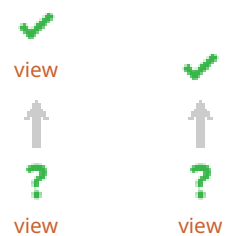 \\ 1. Karl Friston ID, University College London, London, UK \\ 2. Stuart J. Edelstein, Ecole Normale Supérieur, Paris, France \\ 3. László Acsády, Hungarian Academy of \\ Sciences, Budapest, Hungary}

Any reports and responses or comments on the article can be found at the end of the article. 
used to model Darwinian search. The proposed architecture can be used for fast search among stored solutions (by selection) and for evolutionary search when novel candidate solutions are generated in successive iterations. Since all the suggested components are present in advanced nervous systems, we hypothesize that the brain could implement a truly evolutionary combinatorial search system, capable of generating novel variants.

\section{Keywords}

attractor network, autoassociative neural network, evolutionary search, Darwinian dynamics, neurodynamics, learning, problem solving

\section{ingef}

This article is included in the INCF gateway.

Corresponding author: András Szilágyi (and.szilagyi@gmail.com)

Author roles: Szilágyi A: Formal Analysis, Investigation, Methodology, Software, Validation, Writing - Original Draft Preparation; Zachar I: Investigation, Methodology, Software, Validation, Visualization, Writing - Original Draft Preparation, Writing - Review \& Editing; Fedor A: Investigation, Methodology, Validation, Writing - Original Draft Preparation, Writing - Review \& Editing; de Vladar HP: Investigation, Methodology, Software, Visualization, Writing - Original Draft Preparation, Writing - Review \& Editing; Szathmáry E: Conceptualization, Funding Acquisition, Investigation, Project Administration, Supervision, Writing - Original Draft Preparation, Writing - Review \& Editing

Competing interests: No competing interests were disclosed.

Grant information: The research leading to these results has received funding from the European Union Seventh Framework Program (FP7/2007-2013) under grant agreement numbers 308943 (INSIGHT project) and 294332 (EvoEvo project). It was also supported by the project GINOP-2.3.2-15-2016-00057 (In the light of evolution).

The funders had no role in study design, data collection and analysis, decision to publish, or preparation of the manuscript.

Copyright: @ 2017 Szilágyi A et al. This is an open access article distributed under the terms of the Creative Commons Attribution License, which permits unrestricted use, distribution, and reproduction in any medium, provided the original work is properly cited.

How to cite this article: Szilágyi A, Zachar I, Fedor A et al. Breeding novel solutions in the brain: A model of Darwinian neurodynamics [version 2; peer review: 3 approved] F1000Research 2017, 5:2416 https://doi.org/10.12688/f1000research.9630.2

First published: 28 Sep 2016, 5:2416 https://doi.org/10.12688/f1000research.9630.1 


\section{REVISED Amendments from Version 1}

We have answered the questions our Reviewers have asked and also implemented changes to the text to increase its readability and clarity. We have added a glossary (Supplementary File 1) and a Supplementary Figure S1 about the analogy of standard Darwinism and Darwinian neurodynamics, to guide the reader.

See referee reports

\section{Introduction}

The idea that functional selection on a large set of neurons and their connections takes place in the brain during development ${ }^{1-3}$ is now experimentally validated ${ }^{4-7}$. As originally portrayed, this process is only one round of variation generation and selection, even if it requires several years. Evolution by natural selection works differently: variants are generated and then selected in iterative rounds. The field of "Neural Darwinism"1-3 fails to include generation of variants and thus could justifiably be regarded as a misnomer because the process that it describes is not evolutionary in the strict sense ${ }^{8}$. Evidence indicated that the development of the brain is more "constructivist" than pictured by the original selectionist accounts: for example, repeated rounds of neuron addition and loss happen during development ${ }^{10}$. Structural plasticity (synaptic remodelling) is now known to be a lifelong process with implications for memory and learning (e.g. 11,12). The addition and deletion of synapses and neurons takes several hours or days ${ }^{13}$. Our main goal here is to present a proof of principle that bona fide evolutionary dynamics could happen in the brain on a much faster time scale.

Maynard Smith ${ }^{14}$ identified multiplication, inheritance and variability as necessary features of evolution. In genetic evolution the variability operators are mutation and recombination. If there are hereditary traits that affect the survival and/or the fecundity of the units, then in a population of these units, evolution by natural selection can take place. While this characterization qualitatively outlines the algorithmic aspect of evolution ${ }^{15}$, concrete realizations require quantitative conditions too: population size cannot be too small (if it is too small, neutral drift dominates over selection ${ }^{16}$ ) and replication accuracy cannot be too low (if it is too low, hereditary information is $\operatorname{lost}^{17}$ ). Note, that this description says nothing about the nature of the units: they could be genes, organisms, linguistic constructions or anything else.

The proper implementation of an evolutionary process within the nervous system could have major implications for neuroscience and cognition ${ }^{8,18-25}$. A main benefit of neuro-evolutionary dynamics would be that it could harness the parallelism inherent in the nervous system and the redistribution of resources at the same time. The latter process means that hopeless variants are thrown away and are replaced in the "breeding space" by more promising ones $^{8}$. Another important aspect of the process is that it is generative: it could explain where new hypotheses and new policies come from in Bayesian approaches to cognition ${ }^{26,27}$ and reinforcement learning ${ }^{28-30}$, respectively. Bayesian inference and natural selection are analogous $\mathrm{s}^{31,32}$ in that candidate hypotheses in the brain (the prior distribution) represent a population of evolutionary units, which are evaluated, or selected, based on the evidence. There is a mathematical isomorphism between the discrete-time replicator equation and Bayesian updates ${ }^{31}$. The likelihood function is analogous to the fitness function and the posterior distribution to the selected population. Relations like this suggest that Bayesian update could be one of the pillars of "universal Darwinism" 33 . We believe that convincing models for neuro-evolution could empower Bayesian approaches by providing a mechanism to generate candidate hypotheses.

Attractor networks have been used (among others) as models of long-term memory, which are able to complete partial input ${ }^{34,35}$. These networks consist of one layer of units that recurrently connect back to the same layer. The recurrent connections can learn (store) a set of patterns with a Hebbian learning rule. Later, if these patterns or their noisy versions are used to provoke the network, it settles on the original patterns after several rounds of activation updates on the recurrent weights (recall), thus stored patterns act as attractors. It is of high importance that the existence of such networks has been experimentally validated in the visual cortex of awake mice by optogenetic methods ${ }^{36}$. Some versions of the learning rule allow for iterative learning without catastrophic forgetting and enable palimpsest memory. A network with palimpsest memory is able to learn new patterns one-by-one, while sequentially forgetting earlier patterns.

In this paper we describe a model that implements evolution of activation patterns in the brain with the help of attractor networks. We see it as a model of problem solving, which is able to generate new candidate solutions to a problem based on past experiences. Any cognitive problem of the brain is encoded by the activity pattern of neurons. We represent neurons as binary units, being able to continuously maintain or suppress firing. A group of neurons therefore has a binary activation pattern. In our model, the units of evolution are these activation patterns, represented as bitstrings. Attractor neural networks can store patterns stably in form of corresponding attractors and are able to recall them given the appropriate trigger (Figure 1A). This memory allows for heredity, which is indispensable for Darwinian dynamics (in genetic populations memory is the genotype pool). Attractor neural networks can generate new pattern variants in different ways (corresponding to mutation in a genetic system), see below under Discussion. Owing to memory and pattern generation, the possibility of iterated selection over a population of activation patterns becomes feasible. Our approach thus offers a way to implement hereditary dynamics at a faster scale than what could be provided by structural plasticity (cf. 37). This fast-scale dynamics is missing from Edelmanian Neural Darwinism. See the glossary (Supplementary File 1) and Supplementary Figure S1 for more clarification about the analogy between our model and genetic evolution.

The patterns represent candidate hypotheses or candidate solutions to a problem, which are evaluated based on a fitness function that measures their goodness as a solution. The best patterns (those with the highest fitness) are selected and copied (with variation) back to the networks, which in turn generate the next generation of patterns (Figure 1B). 


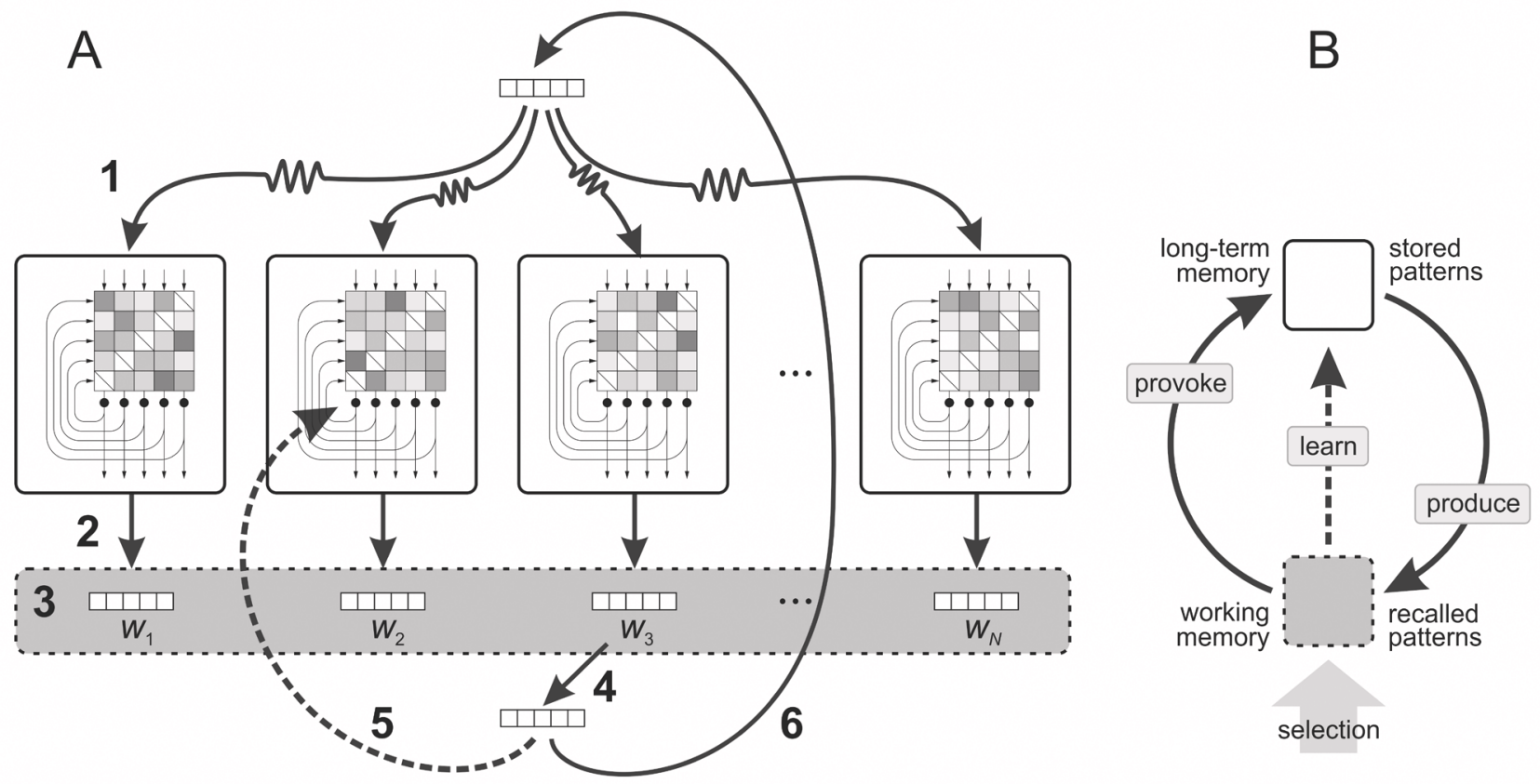

Figure 1. A) Architecture of multiple attractor networks performing Darwinian search. Boxed units are attractor networks. Each network consists of $N$ neurons ( $N=5$ in the figure, represented as black dots). Each neuron receives input from the top (1) and generates output at the bottom (2). Each neuron projects recurrent collaterals to all other neurons (but not to itself), forming thus $N \times(N-1)$ synapses. The weight matrix of the synapses is represented here as a checkerboard-like matrix, where different shades indicate different connection weights. Selection and replication at the population level is as follows: 1) Each network receives a different noisy copy of the input pattern. 2) According to its internal attractor dynamics, each network returns an output pattern. 3) All output patterns are pooled in the implicit working memory (grey box with dashed outline), where they are evaluated and a fitness $w_{i}$ is assigned to the $i^{\text {th }}$ pattern. 4) The best pattern(s) is selected based on fitness. 5) One of the networks is randomly chosen to learn the pattern that was selected, with additional noise (dashed arrow). 6) The selected pattern is copied back to the networks as input to provoke them to generate the next generation of output patterns. B) Lifecycle of candidate solution patterns during a cognitive task. Patterns are stored in the long-term memory as attractors of autoassociative neural networks. When provoked, networks produce output patterns, which are stored in implicit working memory. These patterns are evaluated and selected. Patterns that are good fit to the given cognitive problem can increase their chance to appear in future generations in two possible, non-exclusive ways: 1) selected patterns are used to train some of the networks (learning) and 2) selected patterns are used as inputs for the networks (provoking). The double dynamics of learning and provoking ensures that superior solutions will dominate the system. Erroneous copying of patterns back to the networks for provoking and learning and noisy recall are the sources of variation (like mutations).

Stored patterns (attractors) constitute the long-term memory; output patterns constitute the working memory (Figure 1B). While pattern generation is a simple recall task, which is only able to reproduce previously learnt patterns, the whole system is able to generate new variants due to noisy recall, spurious patterns (see later), noisy copying of patterns, and iterative learning, thus enabling the evolution of novel solutions.

\section{Methods}

Recurrent attractor networks. The basic units in our model are attractor networks. Attractor networks are recurrent neural networks consisting of one layer of units that are potentially fully connected. An attractor neural network produces the same (or highly correlated) output whenever the same input is provided (omitting retraining). The pattern that was learned becomes the attractor point of a new basin of attraction, i.e. it is the prototype pattern that the attractor network should return. Consequently, an attractor with a non-zero sized basin should also return the same output to different input patterns. However, the amount and type of correlation of input patterns that retrieve the same prototype, i.e., the actual structure of the basin of attraction, is hard to assess, let alone visualize. Still, it is safe to assume that most input patterns correlated with the prototype, produce the same output - the prototype itself.

The Hopfield network is a recurrent artificial neural network with binary neurons at nodes and weighted connectivity between nodes, excluding self-connections. According to the usual convention, the two states of binary neurons are +1 and -1 . In our model, a neuron fires $($ state +1$)$ if the total sum of incoming collaterals is greater than 0 . Accordingly, the update rule has the following form:

$$
x_{i}(t+1)=\operatorname{sgn}\left(\sum_{j=1(\neq i)}^{N} w_{i j} x_{j}(t)\right) .
$$


The original Hebbian (covariance) learning rule has the following form (where $\mathrm{m}$ is the index of the patterns):

$$
\begin{gathered}
w_{i j}^{0}=0, \forall i, j \in\{1,2, \ldots, N\}, \\
w_{i j}^{m}=w_{i j}^{m-1}+\frac{1}{N} \xi_{i}^{m} \xi_{j}^{m} .
\end{gathered}
$$

The Hebb rule is both local and incremental. A rule is local if the update of a connection depends only on the information available on either side of the connection (including information coming from other neurons via weighted connections). A rule is incremental if the system does not need information from the previously learnt patterns when learning a new one, thus the update rule uses the present values of the weights and the new pattern. The above update rule performs immediate update of the connection weights ("one shot" process; not a limit process requiring multiple update rounds). The covariance rule has a capacity of $0.14 N^{38}$. However, if the network is trained sequentially, and it reaches its capacity, catastrophic forgetting ensues and the network will be unable to retrieve any of the previously stored patterns, forgetting all it has learnt.

To overcome this problem and to preserve the favorable properties of the covariance rule (one-shot, local and incremental updating) Storkey has introduced a palimpsest learning scheme ${ }^{39}$ as follows:

$$
\begin{aligned}
& w_{i j}^{m}=w_{i j}^{m-1}+\frac{1}{N} \xi_{i}^{m} \xi_{j}^{m}-\frac{1}{N} \xi_{i}^{m} h_{j}^{m}-\frac{1}{N} h_{i}^{m} \xi_{j}^{m} \text { if } i \neq j, \\
& w_{i j}^{m}=0 \text { if } i=j,
\end{aligned}
$$

and

$$
h_{i}^{m}=\sum_{k=1}^{N} w_{i k}^{m-1} \xi_{k}^{m} .
$$

Using the above rule, the memory becomes palimpsest (i.e. new patterns successively replace earlier ones during sequential learning) with a capacity of $C=0.25 \mathrm{~N}$ (for details and proper definition of palimpsest capacity, see 39). Palimpsest memory is essential for our training regime, where we trained the networks sequentially with one pattern at a time.

An interesting feature of some autoassociative neural networks is the appearance of spurious patterns. In some cases, the network converges to a pattern different from any other patterns learnt previously. These spurious patterns can be the linear combination of an odd number of stored patterns:

$$
\xi_{i}^{\text {spur }}= \pm \operatorname{sgn}\left( \pm \xi_{i}^{m_{1}} \pm \xi_{i}^{m_{2}} \ldots \pm \xi_{i}^{m_{s}}\right)
$$

where $S$ is the number of the stored patterns ${ }^{38}$. This effect can be thought of as an effective implementation of a neuronal recombination operator.

In what follows, we will treat a pattern of activations over binary neurons as the unit of selection. In the general case, the adaptive fitness of this pattern may be some complicated function that is contextualized by the current inputs and may or may not be a function of the history of inputs and outputs. To keep things simple, we will just consider the fitness of a pattern in terms of its Hamming distance to some target pattern. This means, we are effectively using selectionist and evolutionary schemes to optimize the connections (and ensuing dynamics) to recover a target pattern.

We present a series of simulations graduating from purely selectionist to evolutionary schemes in the face of a changing environment. In the first set of simulations we preclude variation in transmission over generations to examine the sufficiency of dynamical instabilities in supporting a selective process. This involves taking the outputs of one neural network and using them (after selection) to train another network. In the second set of simulations, we consider evolution proper and the transmission of patterns from generation to generation. Here, the patterns that are transmitted are subject to mutations (and selection) to illustrate the efficiency with which optimal patterns (those with high fitness) emerge.

Selection. For the selection experiment, we used $N_{A}=20$ structurally identical attractor networks, each consisting of $N=200$ neurons. These networks were initially trained with random patterns plus a special pattern for each with Storkey's palimpsest learning rule. The 20 special training patterns were as follows. The worst special pattern was the uniform -1 , the best special pattern was the uniform +1 . Intermediate special patterns had increasing number of +1 -s from the left. Fitness was measured as the relative Hamming similarity from the globally best target $O_{\text {target }}$ (i.e. the proportion of $+1-\mathrm{s}$ in the pattern). The worst special pattern was presented only to network \#1, the second worst to \#2, etc., while the best special pattern (which was the target pattern) was presented to network \#20. In this scenario, no further training occurred (i.e., the dashed arrows on Figure 1 are not there). Assuming that the attractor basins of these patterns overlap among networks (Figure 2A) the output of one network will be the cue to trigger one or more close special patterns in other networks. The special patterns ensure that there exists a search trajectory leading from the worst to the best pattern, fitness-wise. Starting from any arbitrary initial pattern, if any of the special patterns gets triggered at any time, the system can quickly converge to the optimum - providing the output of each network is delivered to the appropriate network that successively converges on the global optimum.

After initial training, each network received the same random input and generated an output according to its internal attractor dynamics. The output population was evaluated and the best output $O_{\text {best }}$ was selected based on its fitness. Noisy copies (with $\mu_{I}$, where $\mu$ is the per-bit mutation probability) of $O_{\text {best }}$ were redistributed for each network as new input for the next generation. These steps were iterated until fitness reached the theoretical optimum (i.e. the system found special pattern \#20). The crucial assumption for selection to work is continuity, namely the possibility that the output of one attractor of one network could fall in a different attractor basin of another network returning an output that is closer to the global optimum than the input was (see Figure 1 and Figure 2).

Evolutionary optimization on a single-peak landscape. In contrast to purely selective dynamics, in the evolutionary experiment, 


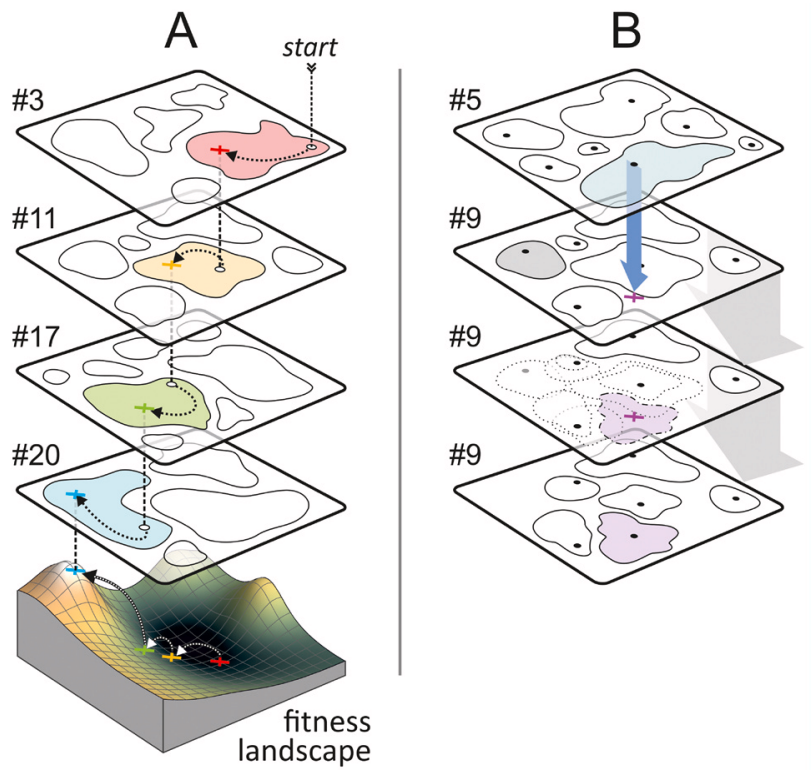

Figure 2. Schematics of attractor networks searching for the global optimum. A) Four time steps of selection, from top to bottom. At each step, we only show the network that produces the best output (numbered); the rest of the networks are not depicted. In each time step the networks are provoked by a new pattern that was selected from the previous generation of patterns. Different attractor networks partition the pattern-space differently: blobs inside networks represent basins of attraction. At start, the topmost network (\#3) is provoked with an input pattern. It then returns the center of the attractor basin which is triggered by the input. When the output of this network is forwarded as input to the next network (\#11), there is a chance that the new attractor basin has a center that is closer to the global optimum. If there is a continuity of overlapping attractor basins through the networks from the initial pattern (top) to the global optimum (bottom), then the system can find the global optimum even without learning. B) Learning in attractor networks. Network \#5, when provoked, returns an output pattern that is used to train network \#9 (blue arrow). As the network learns the new pattern, the palimpsest memory discards an earlier attractor (with the gray basin), a new basin (purple shape) forms around the new prototype (purple $x$ ) and possibly many other basins are modified (basins with dotted outlines). Black dots indicate attractor prototypes (i.e. learnt patterns). With learning, successful patterns could spread in the population of networks. Furthermore, if the transmission of patterns between networks is noisy a network might learn a slightly different version of the pattern, new variation is introduced to the system above and beyond the standing variation. This allows the system to find the global optimum even if it was not used to pre-train any network. The gray arrow in the background indicates the timeline of network \#9.

networks could learn new patterns during the search process. At the start, each network was trained with a different set of random patterns. The fitness of a pattern is defined as the relative (per bit) Hamming similarity between the given pattern and an arbitrarily set globally best target pattern $O_{\text {tarret }}$. The selection process for the actual best output $O_{\text {best }}$ and redistribution of its noisy copies (with $\mu_{I}=0.005$ ) for input was the same as before. Most importantly, the mutated versions (with $\mu_{T}=0.01$ ) of $O_{\text {best }}$ were also used for retraining $N_{T}$ different networks in each generation (see Figure 1): this forms the basis for the Darwinian evolutionary search over attractor networks, as it allows for replication with variation of (learnt) patterns over networks (thin lines in Figure 3).

We have also analyzed how mutation rates affect the speed of evolution. We measured the number of generations needed for the system to find the globally best target pattern. The previously introduced parameters were used and the mutation rates were varied between $10^{-4}$ and 0.01 . We ran each simulation 100 times with different random seeds and measured the average number of generations and standard deviation. We found that only $\mu_{l}$ affects the speed of evolution with an almost constant minimum in a wide range between approx. 0.003-0.007. Mutation rates lower than 0.003 or higher than 0.007 result in rapidly growing number of generations necessary for the system to find the optimum. $\mu_{T}$ does not affect the speed of the evolution considerably (data not shown).

We have compared the search behavior of our system of attractor networks with a simpler model. In this model networks were represented as abstract storage units, which could store exactly $C_{f x}$ patterns $\left(C_{f x}\right.$ was set to be close to the actual capacity of networks). When such a storage unit receives an input pattern it simply returns the closest (in Hamming distance) of its stored patterns as output, with additional noise $\left(\mu_{O}=0.001\right)$. The units simulate the almost perfect recall property of attractor networks and effectively approximate attractor behavior. We compared evolution in this simple model with evolution in the system of attractor networks (thick and thin lines in Figure 3).

Optimization in a changing environment. In order to test the effect of memory on successive search, we have implemented a periodically changing selective environment, i.e., we periodically changed the fitness function. The environment alternated between $E_{1}$ and $E_{2}$, with a stable period length of $T_{E}=2000$. Each environmental change reset the global optimum: for this scenario, we assumed a uniform +1 sequence for $E_{1}$ and its inverse, uniform -1 for $E_{2}$ as global optima, and used the relative Hamming similarity as a fitness measure.

In the first phase of the simulation, networks were allowed to learn in each environment for a total of $T_{\text {nolearn }}=12000$ generations (three periods per environments). Afterwards, learning was turned off to test the effect of memory. To make sure that the optimal pattern was not simply carried over as an output pattern from the previous environment but was recalled from memory, the input patterns were set to random patterns (instead of inheriting the previous output population) at the start of each new environmental period after $T_{\text {nolearn }}$. This ensures that the population could only maintain high fitness afterwards in an environment if the optimum was stored and could be successfully recalled (see Figure 4). In order to assess the memory of a network, we also measured the distance between the actual best output of the population and the closest one of the set of previously learnt patterns within the same network (as different networks have different training history). A small distance indicates that the network outputs a learnt pattern from memory (i.e. recalls it) instead of a spurious pattern.

For this scenario, we introduced a different selection method (also used in the next section). Each network in the population 


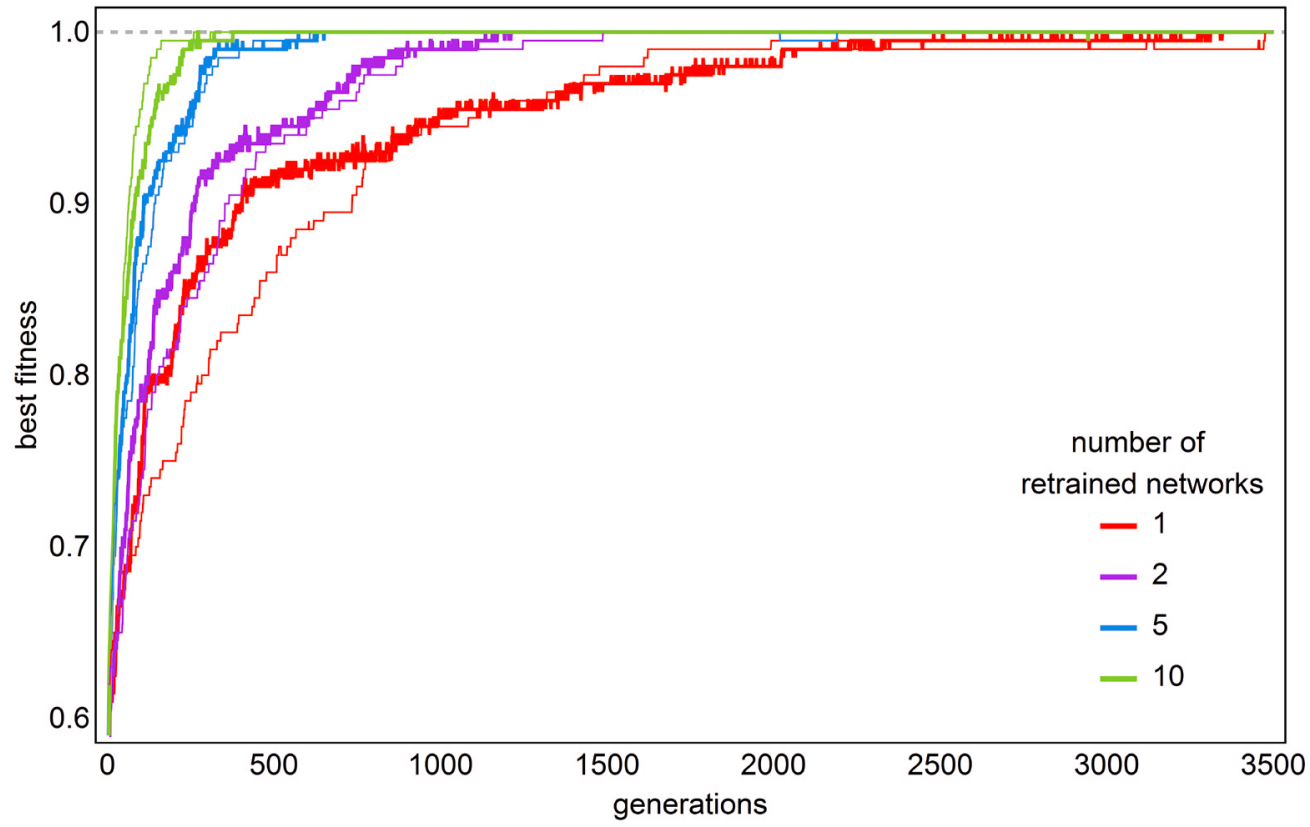

Figure 3. The effect of retraining on the speed of evolution. Lines represent the evolution in four different populations, where a different number of networks were retrained. Each population consisted of 10 networks (see the rest of the parameters under the Methods section). Thin lines: stochastic attractor dynamics; thick lines: simulated attractor dynamics (abstract networks always return the stored attractor prototype that is closest to the actual input, with 0.001 per bit probability noise; capacity to store $C_{f i x}=30$ patterns, $\mu_{0}=0.002$ ). Parameters: $N=200, N_{A}=20, \mu_{T}=0.01, \mu_{1}=0.005$, elitist selection, keeping the best one only from each output generation; random networks are selected for retraining (never the same in a given generation). Fitness is the relative Hamming similarity to the global optimum.

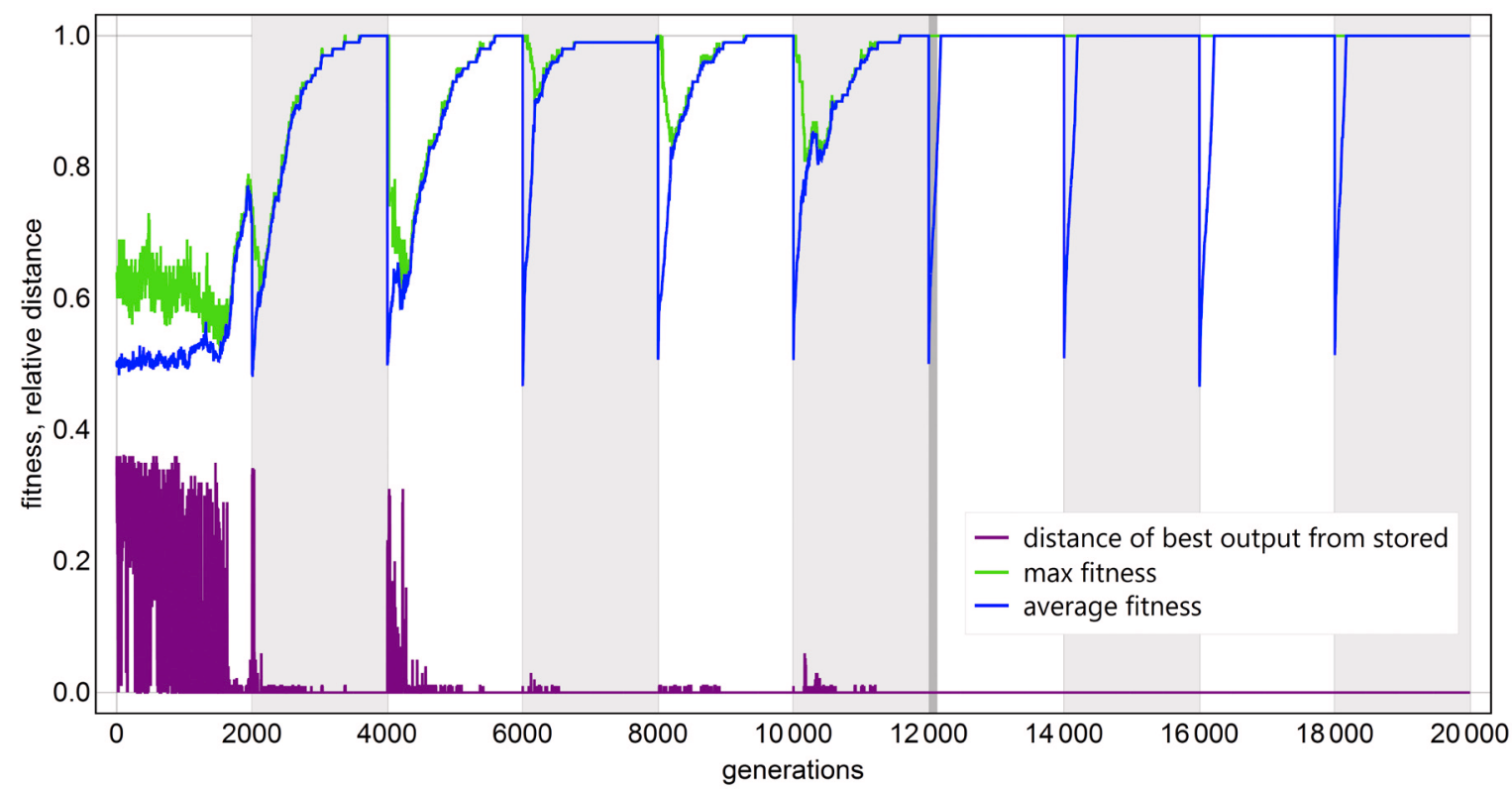

Figure 4. Fitness and recall accuracy over periodically alternating environments. Blue: average fitness; green: best fitness; purple: distance of the best output of the population from the closest one stored in memory (for details, see main text). Grey and white backgrounds represent the changing environment: We alternated two global optimums at every $2000^{\text {th }}$ generation. After the $12000^{\text {th }}$ generation, we turned off learning (thick vertical line) and set the input to random patterns after each changing of the environment. Parameters: $N_{A}=100$, $N=100, N_{T}=40$, fitness is the relative Hamming similarity to the actual optimum. 
produces an output according to its internal attractor dynamics and the input it received from the previous generation. From all output sequences one was randomly chosen and mutated $\left(\mu_{R}=1 / N\right.$ per bit mutation rate). If the mutant had a higher fitness than the worst of the output pool, the worst pattern was replaced by it (elimination of the worst). Furthermore, the superior mutant was used to train $N_{T}$ number of different networks. Lastly, the resulting output population is shuffled and fed to the networks as input in the next generation (except when the environment changes and input is reset externally).

Optimization on a difficult landscape. To investigate the applicability of this optimization process, we adopted a complex, deceptive landscape with scalable correlation, and also modified the selection algorithm introduced above. We used the general building-block fitness (GBBF) function of Watson and Jansen ${ }^{40}$. According to the GBBF function, each sequence of length $N$ is partitioned into blocks of uniform length $P$, so that $N=P B$ $\left(P, B \in \mathbf{Z}^{+}\right.$) where $B$ is the number of blocks. For each block, $L$ arbitrarily chosen subsequences are designated as local optima, with randomly chosen but higher-than-average subfitness values. The overall fitness $F(G)$ of a pattern $G$ ("genotype") is as follows:

$$
\begin{gathered}
F(G)=\sum_{i=1}^{B} f\left(g_{i}\right), \\
f\left(g_{i}\right)=\sum_{j=1}^{L} c\left(g_{i}, t_{j}\right), \\
c\left(g, t_{j}\right)=\left\{\begin{array}{cc}
w_{j}, & \text { if } d\left(g, t_{j}\right)=0 \\
\left(1+d\left(g, t_{j}\right)\right)^{-1}, & \text { otherwise }
\end{array},\right.
\end{gathered}
$$

where $f\left(g_{i}\right)$ is the fitness contribution of the ith block in the pattern, $t_{j}$ is the $j^{\text {th }}$ local optimum of length $P$ (all $L$ different optima are the same for each block in our experiments) with subfitness value $w_{j}>1$, and $d$ is the Hamming distance. Consequently, this landscape has many local optima, a single global optimum and a highly structured topology. Furthermore, since there are no nonlocal effects of blocks, each block can be optimized independently, favoring a metapopulation search.

Accordingly, in this experiment, we introduced multiple populations of attractor networks. Each population of $N_{A}$ attractor neural networks forms a deme and $N_{D}$ demes are arranged in a 2D square lattice of Moore neighborhood (all of the eight surrounding demes are considered neighbors). Demes might accept output sequences from neighboring demes with a low probability $p_{\text {migr }}$ per selection event; this slow exchange of patterns can provide the necessary extra variability for recombination. These demes correspond to the groups of columns in the brain.

Networks in the deme are the same as those used in previous experiments. However, selection is modeled in a different way, similar to the selective dynamics outlined in 40. In turn, we only give a brief description. Given a deme, each network produces an output according to its internal attractor dynamics and the input it received from the previous generation. Output sequences are pooled and either one or two is randomly chosen for mutation or recombination, respectively (i.e. no elitist selection). With probability $p_{\text {rec }}$, two-point recombination is performed in the two selected partners, with $1-p_{\text {rec }}$ probability, a single selected sequence is mutated, with $\mu_{R}=1 / N$ per bit mutation rate. With $p_{\text {migr }}$ probability, the recombinant partner is chosen from another neighboring deme instead of the focal one. Next, the output(s) of recombination or mutation are calculated: if the resulting sequence (any of the two recombinants or the mutant) has a higher fitness than the worst of the output pool, it is replaced by the better one (elimination of the worst). Furthermore, the superior mutant or recombinant was used to train $N_{T}$ number of different networks within the deme. Lastly, the resulting output population is shuffled and fed to the networks as input in the next generation. Each deme is updated in turn according to the outlined method; a full update of all networks in all demes constitutes a generation (i.e. a single time step).

The GBBF landscape was set up identically to the test case in 40, as follows. For each block uniformly, two target sequences of length $P, T_{1}$ and $T_{2}$, were appointed. $T_{1}$ is the uniform plusone sequence $T_{1}=\{+1\}^{P}$ and $T_{2}$ is alternating between -1 and $+1\left(T_{2}=\{-1,+1\}^{P / 2}\right)$. According to the fitness rule (Equation 5Equation 6 in 40 and Equation 1-Equation 3 above), the best subfitness of each block in a sequence can be calculated and the sum of all the subfitness values is the fitness of the global optimum sequence. Thus for sake of simplicity, we used relative fitness values with the global optimum (the uniform +1 sequence) having maximal fitness 1 . The sequence(s) with lowest fitness always have a nonzero value.

The source code of all models and data presented in this paper is freely available as a supplement to this paper.

\section{Results}

Selection. We should distinguish between two processes: (i) search without learning among the stored patterns to find the best available solution (i.e., selection without step 5 on Figure 1A), and (ii) search with learning: retraining one or more networks with the selected and mutated patterns (Figure 1A with step 5). The first is a purely selectionist approach because it cannot generate heritable variants, while the second implements Darwinian evolution because learning changes the output behavior of the networks, thus they generate new patterns. First, we analyze the strictly selectionist version, and then the evolutionary version of the model.

In the selectionist version we pre-trained each network with a random set of patterns (excluding the target pattern) and started by provoking them with a different random input. Each network produced an output pattern according to its own attractors and then the best pattern was selected. This pattern was used in turn to provoke the networks in the next generation, and so on. This search has found among all the available stored (pre-trained) patterns the one with the highest fitness; it could not find the global optimum, as the networks were not pre-trained with it and there was no way for new variants to survive in this simulation. 
Next, we specifically composed the sets of pre-training patterns: each network was pre-trained with random patterns as before but also with one special pattern. This set of special patterns (in which individual patterns can be ordered according to gradually increasing fitness values) delineate a route to the optimum through overlapping basins of attractors in different networks (see Figure 2A) so that we can test whether in this simplified case the algorithm converges quickly to the optimum. The first population was initiated with the special pattern that was farthest from the optimum. We have found that the selected output gets closer to the optimum in each generation, but the optimization process is saltatory: it skips over many intermediate neighboring special patterns (and thus networks). This is due to the fact that attractor basins of neighboring special patterns were highly overlapping. For example, in Figure 2A, the stored special pattern of network \#3 is in the basins of stored special patterns of networks \#4-\#11, and since the stored pattern of network \#11 is closest to the optimum, networks \#4-\#10 were skipped. A typical sequence of networks generating the actual best output is: \#3, \#11, \#17 and \#20 (of 20 networks; for actual parameters, see Figure 2A).

Evolution. Learning new patterns as attractors (Figure 2B) allows networks to adapt to the problem and perform evolutionary search. The results of the evolutionary experiments clearly prove that a population of attractor networks can implement evolutionary search in problem spaces of different complexity (i.e. different levels of correlation and deceptiveness).

Evolution on a simple fitness landscape. In this scenario, neither the global optimum nor a route toward it is assumed to pre-exist in the system as in the selectionist experiments: networks are pretrained only with random patterns. Even under these stringent conditions, we have found that the system can converge to the global optimum, and this convergence is robust against a wide range of mutation rates. Our simplified abstract model, which always returns the stored prototype that is closest to the input, behaves qualitatively the same way (see Figure 3 ). The speed of convergence to the optimum is mainly affected by the number of retrained networks (Figure 3): as we increase the number of networks that are retrained we find a faster fitness increase, albeit with diminishing returns. Mutation has an optimal range in terms of the speed of evolution. On one hand, if mutation rate is too low evolution slows down, because there is not enough variation among patterns. On the other hand, if mutation rate is too high it hinders evolution as the offspring is too dissimilar to the parent and cannot exploit the attractor property of the system. When mutation rate is zero, the source of variation is only the probabilistic input-output behavior of the networks due to their asynchronous update and the appearance of spurious patterns when the input is too far from the stored patterns.

While the attractor networks have memory, due to the monotonic, single-peak nature of the fitness landscape there is no need to use it: the system works almost equally well if the networks only store the last training pattern (i.e., weights are deleted before each learning event). Next, we present experiments where both the attractor property and the palimpsest memory of the networks are used.
Evolution in a changing environment. In this experiment we alternated two environments: in every 2000th generation the target pattern (the optimum), against which fitness was measured, was changed. From an evolutionary point of view, this can be perceived as a changing environment, whereas from a cognitive point of view, this procedure simulates changing task demands. Figure 4 shows that the system found and learnt the optima of each of the two environments separately. Then, after generation 12000, we switched off learning. The fact that networks are nevertheless able to recall the target pattern right after the environmental change proves that they use previously stored memories. After we switched off learning, we used random patterns to provoke networks at the first generation of each new environment. A single network that can recall the optimum from the random input is enough to produce a correct output that is amplified by selection for the next generational input, ultimately saturating the population with optimal patterns. This experiment effectively proves that a system of attractor networks can reliably recall earlier stored solution patterns, therefore solving the problem faster in an alternating environment than a system without long-term memory.

Evolution on a difficult fitness landscape. The previous evolutionary experiment (where search was on a single-peak fitness landscape with a single population of networks) is a proof of principle of the effectiveness of our evolutionary algorithm. In order to assess the capacity of population search of attractor networks, we introduce a considerably harder fitness landscape with higher dimensionality, where the deceptiveness of the problem can be tuned. The GBBF fitness landscape of 40 provides a method to easily generate scalable and complex landscapes with many deceptive local optima. The complexity of the problem requires the introduction of multiple interacting populations of networks. Though explicit spatial arrangement of the networks is not required to solve the problem, we have nevertheless included it in our implementation to imitate real spatial arrangement of neurons in the brain. Locality allows the exchange of information among neighboring populations (i.e. recombination) that is essential to solve the GBBF problem (or similar deceptive problems) in a reasonable time.

We have investigated the performance of search in a metapopulation with different problem sizes (pattern lengths; see Figure 5). Results indicate that despite the vastness of the search space, the metapopulation is always able to converge to the global optimum, given enough time. The most complex landscape of 100-bit patterns is of size $2^{100}$ with one global optimum and a huge number of local optima. The metapopulation consists of $10^{5}$ neurons (100 populations of 10 networks each with 100 neurons per network) and can find the single global optimum in $\sim 10^{4}$ time steps. The limit of further increasing the problem size is in the computational capacity of our resources.

\section{Discussion}

Summary of results. Attractor networks can be used to implement selection, replication and evolutionary search. As a proof of principle, we showed that attractor networks find the global 


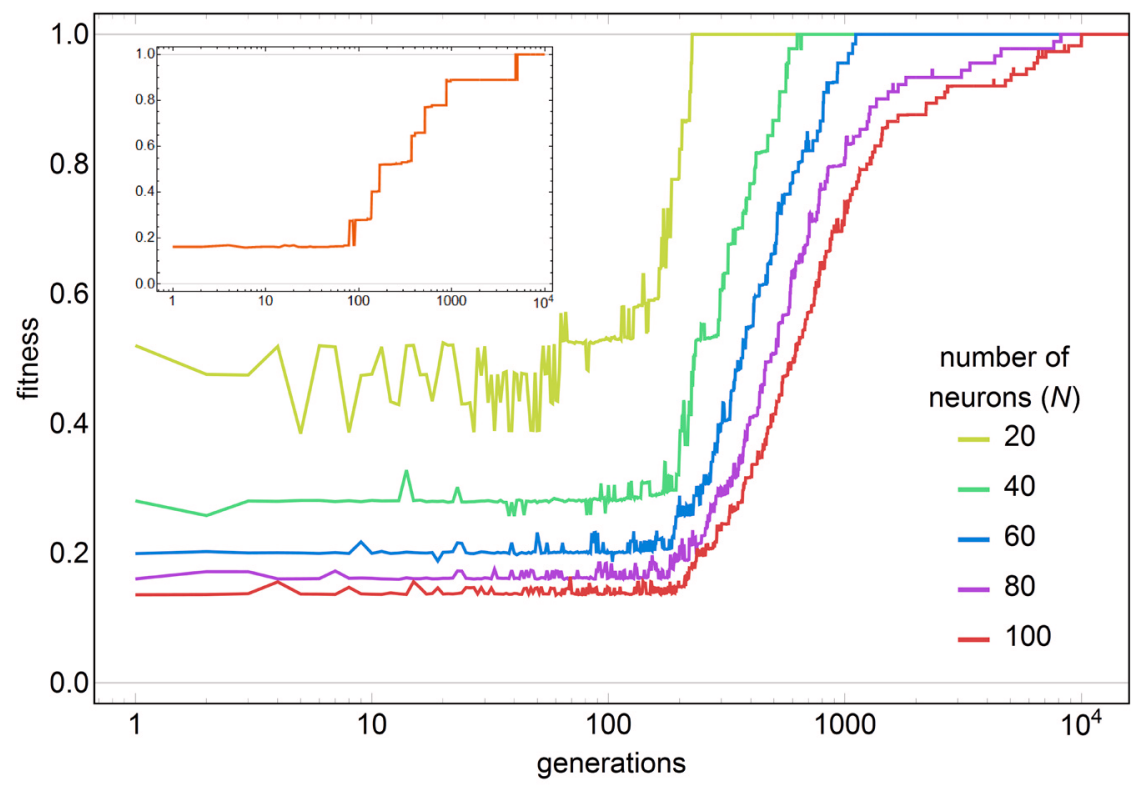

Figure 5. Convergence of the actual best fitness of the metapopulation with increasing problem size $\boldsymbol{N}$ on general building block function landscape. Each curve is an average of 10 independent iterations. $N_{P}=10 \times 10$ demes, $N_{A}=10$ networks per deme, $N$ neurons per network, patterns of length $N$ are partitioned to blocks of size $P=10$ ( $B=N / P$ blocks per pattern), $p_{\text {rec }}=0.1, \mu_{R}=1 / N, p_{\text {migr }}=0.004, N_{T}=5$. Note the logarithmic $x$ axis. Inset: single simulation at $N=80, B=8$ (other parameters are the same). Plateaus roughly correspond to more and more blocks being optimized by finding the best subsequence on the building-block fitness landscape.

optimum in a purely selectionist model (i.e. without learning) if they are pre-trained with attractors that overlap in their basins and lead to the optimum. The population can effectively select over the standing variation of all stored patterns and find the trajectory to the (single) peak of the fitness landscape (see Figure 2B). Furthermore, if learning is allowed during search, the relative frequency of good patterns (those closer to the optimum) can be increased by re-training networks with such patterns, so that they are stored in the long-term memory in more copies (Figure 3). Overwriting an older memory trace with a new pattern corresponds to a copying operation, potentially with mutations. A particularly interesting aspect of a population of attractor networks under the given coupling is that even if learning is not erroneous, the Lamarckian nature of inheritance of patterns (as output $\rightarrow$ memory $\rightarrow$ output; see Figure 1B) means that there is room for heritable variation to emerge at other stages of the cycle, thus implementing Darwinian dynamics.

The explicit benefit of memory manifests in a periodically changing environment. In a single, stable environment, memory is not very useful because the attractor property acts against exploring variation, and networks might be even slower than gradient hillclimbers (i.e. searchers who generate mutant output blindly and only take a step on the landscape if the output is better than the input). However, in a periodically changing environment, attractor networks with memory are able to recall the actual global optimum if they have already encountered the given environment in the past and stored its optimum; hill-climbers or naïve attractor networks lacking the necessary memory would have to perform the search all over again. Attractor network can recall the appropriate pattern even if there is no initial cue for the population to know which environment it is in. A network with memory can complete a partial cue and thus can recall the global optimum. After learning has ceased, it is enough to only have a few networks in the population that can recall the optimum from random cues that are accidentally close to the actual optimum (maximal fitness is immediately 1 in the population, see green curve in Figure 4). Selection then simply amplifies these optimal output patterns in the population (as output is fed back as input in the next generation) until all networks receive optimal patterns as input. At that point, average fitness also reaches the maximum (Figure 4, blue curve). A network without memory would have to search for the new optima in each environment over and over again, finding the whole uphill path on the landscape.

We also proved that a metapopulation of attractor networks can successfully optimize on a complex, correlated landscape of higher dimensionality. This is a notoriously hard optimization problem (cf. 40) as hill-climbers can easily get stuck in deceptive local optima. The spatial organization of attractor networks resembles the spatial organization of neural structures of the cortex and it allows parallel optimization of subproblems. By this independent optimization of solution parts, local populations can exchange successful partial solutions with each other and form recombinants that are necessary to solve such complex problems in reasonable time.

Evolutionary context. We have chosen attractor networks to demonstrate Darwinian neurodynamics because (i) the search for existing solutions uses the same architecture for generating, testing and storing novel ones; (ii) stored patterns help evolutionary 
search by readily employing related past (learnt) experience, and (iii) the particular choice of Storkey's model naturally results in some new recombinant patterns. This is an important point because, as we know from population genetics ${ }^{41}$, recombination speeds up the response to selection by creating new variants ${ }^{42}$. For a non-exhaustive mapping between population genetics ad Darwinian neurodynamics (see Supplementary File 1 and Supplementary Figure S1).

Our choice of implementation of attractor networks, following Storkey's model ${ }^{39}$, is based on three important aspects: (i) it has palimpsest memory, so that it can learn new and forget old patterns without catastrophic forgetting, as happens in Hopfield and other networks; (ii) its attractors are roughly of equal size and are well-structured according to a Hamming distance measure, and (iii) unlike most other neural networks, it is able to store correlated patterns. The downside is that these networks require full connectivity, which is neuronally unrealistic. However, its functional properties reflect well what we know of long-term memory in the brain, which is enough for a proof or principle of an evolutionary implementation of neuronal function. To our knowledge no model exists in the literature that could satisfy all requirements above and that, at the same time, work with diluted connectivity ${ }^{43}$.

It is important to clarify that the units of evolution in our proposed Darwinian neurodynamics system are the activation patterns: they are copied potentially with errors, selected and amplified. However, patterns live in two stages: in the working memory and in the long-term memory (Figure 1). This implies different inheritance methods (routes to pass on information) from what is expected in a purely genetic system. Changed attractor prototypes imply changed outputs, just like a mutated genotype implies a different phenotype. However, in our proposed system, changes made to output patterns (by mutation) can also be "inherited" by the stored attractor prototypes via learning. Furthermore, there is another difference in the dynamics, explained in turn.

Darwinian evolution is often described as a parallel, distributed search on a fitness landscape ${ }^{8}$. The population, as an amoeba, climbs the peaks of the landscape via iterative replication and selection in successive generations. The attractors, however, impose a different mode of evolution, because they simply return the prototype pattern closest to the input, even if it is less fit than the input pattern itself. Consequently, attractor networks work against variability and slow down hill-climbing. However, attractor networks resist fitness increase only half of the time on average; the other half of the time they effectively push inferior patterns uphill in the fitness landscape at a speed much higher than that expected for the same (reduced) amount of genetic variation. Consequently, attractor networks can facilitate evolution ${ }^{21}$.

We stress the importance of evolutionary combinatorial search. In cases where $a b$ initio calculation of molecular functionality is impossible, artificial selection on genetic variants has proven to be an extremely useful method to generate molecular solution to functional problems, as experiments on the generation of catalytic RNA molecules (ribozymes) illustrate (see 44 for a recent review). By the same token when a brute force numerical calculation of a combinatorial functionality problem is impossible for the brain, given the adequate architecture it could (and we suggest it does) use an evolutionary search mechanism, as shown in this paper.

Implementation in the brain. It is of primary importance that all the components in our 'algorithmic diagram' (Figure 1) can be implemented by mechanisms known in the brain. It is likely that the cortical hypercolumn ${ }^{45}$ behaves like an (at least one) attractor network. The reciprocal connections between the long-term and working memory networks are assumed to be like those in Figure 3 in 46 . We propose that, first, the reinforcing signal from the basal ganglia via the thalamus keeps active the good candidate pattern solutions in the rewarded auto-associative network and, second, that the latter sends a copy of the active pattern to (unconscious) working memory for eventual redistribution. When there is an increase in the quality of a solution (fitness increase) or when a local or global optimum is reached, the central executive elicits the transmission of a copy of the solution to the conscious memory ${ }^{47}$.

Our proposed mechanism relies on information transmission of patterns between cortical groups and relevant subcortical structures with variation, and in this way it differs from all previous models. A discussion of timescales is in order. Without learning newly generated variants, the selective mode would require about the same time as suggested by 48 as the "cognitive cycle" (based on data), but without perception at the beginning of the cycle, i.e. it would be in the $160-310 \mathrm{~ms}$ range. In the evolutionary mode, learning of new variants is required which would take more time. A conservative estimate for the reliable expression of changed synaptic weights is between seconds to minutes ${ }^{49,50}$. The second scale would allow several dozen cycles/generations per minute-a very good number in artificial selection experiments. A more accurate estimate of timing will require a fully neuronal model of our proposed mechanism.

Copying of information is necessary for evolutionary processes: this is where previous approaches ${ }^{22-24,51}$ have been too artificial. There are four well known instances where scholars invoke copying of information in the brain: (i) the transfer of information from short to long-term memory ${ }^{35,52,53}$, (ii) the transfer of information from specialized cortical areas to working memory ${ }^{54}$ or to the global workspace ${ }^{55,56}$ and, finally (iii) the possible copying of patterns from working memory to conscious processing ${ }^{57}$. Undoubtedly, all these approaches require accurate information transfer between different brain components ${ }^{58}$.

In the context of Darwinian neurodynamics, inheritance is replaced by learning. De Vladar and Szathmáry ${ }^{21}$ showed that the rate of "mutation" in spiking diminishes as there is convergence to the optimum. In other words, as the population of attractors approaches the solution, the rates of mutation decrease, since the need to generate new hypotheses decreases. This type of adaptive mutation rate results in what is known as "evolvability" 59 and in Darwinian neurodynamics it comes for free: while selection ensures that more accurate hypotheses are implemented, learning through the strengthening of connections between simultaneously active neurons decreases the rate of variability. Hence, the crucial effect in neurodynamics comes from the built-in mechanism that effectively 
implements second-order selection by adapting the mutation rate according to the quality of the standing hypotheses.

There are three sources of variation in our system: (i) due to the finite size of our networks and asynchronous update of the neurons, the output patterns show some variation even if a network is repeatedly provoked by the same input pattern (noisy recall), (ii) acknowledging the noisiness of neuronal transmission we introduce variation akin to mutations when patterns are transmitted among the blocks of the model (we mutate the patterns with mutation rate $\mu$ ), and finally (iii) we have realized that "spurious patterns" 38 emerge as by-products of previous training patterns and they might act as (new) recombinants of learnt patterns that facilitate the evolutionary search.

The non-conscious or implicit working memory, which has received considerable attention lately ${ }^{47,57}$ is crucial for our proposed mechanism. Irrespective of whether working memory overlaps with the conscious domain ${ }^{60}$ or not $^{57}$ (in the latter case a 'conscious copy' must be sent from working memory to conscious access), the important factor is that the bound on the number of patterns that can be held in the unconscious part of the working memory is larger than that of the conscious working memory ${ }^{60}$. In other words, our mechanism suggests that the total storage capacity of the unconscious network population is much higher than that of the conscious one. Crucially, there is support for this requirement: there is evidence that the central executive function of working memory is not restricted to the conscious domain either ${ }^{47}$. The relatively large capacity of (unconscious) working memory can hold not one, but several patterns selected by the cortex-striatum-basal ganglia loop. This type of selection can be realized by a winner-share-all (WSA) mechanism ${ }^{61}$.

The latter point requires special attention. The reader is referred to the recent review by 62 on models of action selection and reinforcement learning. We wish to make a few critical points in this regard. First, as we are considering problem solving that unfolds its capacity online, there is no reason to select one pattern, since the interim patterns are not alternative actions but only candidate solutions. They can be turned into actions sometimes during, or only at the very end, of the evolutionary search. Weak lateral inhibition within the evaluation mechanism enhances value differences in selection, but a single winner is not selected ${ }^{61,62}$. Second, parallelism of the evolutionary approach loses considerable power if the evaluations are not done in parallel, and if poor solutions cannot be replaced by better solutions in the storage. (In a subsequent study we shall show that the number of parallel evaluations are allowed to be considerably smaller than population size but also that purely serial evaluation of candidates is a killer). Third, it is perfectly possible that the WSA part is implemented by the cortex rather than the striatum (cf. 62): we are agnostic on this point for the time being. (Admittedly that option would require a different version of the full model). Fourth, we maintain that parallel survival of a number of candidates should happen, and the mechanism for this might have evolved with selection for complex (offline) problem solving. Fifth, it is well possible that WSA is gradually reduced towards WTA (one winner takes all) during evolutionary search in the brain: this would also guard against premature convergence early and fast convergence towards the end of the search.

To sum up, we have seen that a process analogous to natural selection can be rendered into a neuronal model employing known neurophysiological mechanisms. Now we discuss relations to some other publications and outline future work.

Related work. Several examples show that evolution with neurodynamics can be more powerful than either of the components alone. Fernando et al..$^{25}$ proved that the path evolution algorithm - which includes both elements of structural plasticity $^{63,64}$ and iterative generation of variation - is more powerful in several regards than classical genetic algorithms. Fernando et al. have also shown that replication combined with Hebbian learning is more powerful than classical natural selection in a model of mechanistic copying of binary activity ${ }^{22}$. De Vladar and Szathmáry provided proof that the synergy between selection and learning results in increased evolvability; also they pointed out that synaptic plasticity helps escaping impasses and build circuits that are tailored to the problem ${ }^{21}$. Finally, in a recent model Fernando and his colleagues have used autoencoders for the generation of "neuronal genotypes" ${ }^{6}$. Since autoencoders produce compressed representations of the input, we expect them to successfully replace the identity function (i.e. bit by bit copying, as in DNA replication). Indeed, applying this neural component within the context of a genetic algorithm turned out to be rewarding.

Unless the envisaged information transfer is accurate enough in space and time, the evolutionary dynamics breaks down. Similar to genetic evolution, where the error threshold ${ }^{17}$ had to be raised before long informative genomes could arise by evolving adaptations for more accurate copying ${ }^{66}$, in the neuronal context the element of accuracy was raised by Adams ${ }^{18}$. In his "Hebb and Darwin" paper Adams talks about synaptic replication and synaptic mutation as important ingredients for a Darwinian view of the brain. Synaptic replication means either the strengthening of an existing synapse, or the making of a new synapse between two neurons that already have one synapse between them. Adams' is an important insight: evolutionary dynamics does not need copying for selection (scoring or strengthening is enough), but it needs copying with errors to test the new variants against the others. Synaptic mutation happens when a neuron grows a synapse towards a neighboring neuron with which previously it had no contact. Interestingly, these thoughts preceded the burst of interest in structural synaptic plasticity (SSP, ${ }^{63,64}$ ). Following his expansion-renormalization model for $\operatorname{SSP}^{67}$ Kilgard observes that when SSP is used for learning something new, this could be regarded as a Darwinian mechanism ${ }^{37}$, as it generates and tests variations in successive rounds, based on what is already there (unlike the original models of "neural Darwinism"). Kilgard's mechanism has not been formalized yet (although see 21), but the path evolution mode ${ }^{25}$ bears some relationship to it.

We share the view of Eliasmith ${ }^{54}$ that the cortex/basal ganglia/ thalamus/cortex loop plays a crucial role not only in elementary action selection but also in symbolic reasoning. We conjecture that non-primate animals (in particular mammals and birds) employ the 
same (or at least an analogous) loop in order to retrieve old and to innovate new solutions, in a similar way as we have shown using our elementary model.

Another view to which we feel strongly related to is that of Bayesian models that advocate "theory learning as stochastic search in a language of thought" 27 . We are reasonably confident that we have found a candidate mechanism for the search process. If true, the rugged learning landscape in Figure 3 of 27 can be directly interpreted as the fitness landscape of our neuroevolutionary model. A task for the future is to work out the explicit relations in detail. We note again the formal link between Bayesian inference and evolutionary selection ${ }^{31,32}$ mentioned in the Introduction. This has further implications for the Bayesian brain, which indicate that our neuro-evolutionary framework is not only a metaphor, but works in a way that is at least equivalent to an evolutionary search. From the Bayesian perspective, the brain weights a series of candidate hypotheses according to their likelihood. From the evolutionary perspective, this update is interpreted precisely as the action of selection on the standing round of hypotheses, with the next round or generation given by the posterior probability of these hypotheses. Moreover, the selective likelihood has been shown in previous models of population genetics to be analogous to a free energy function that is minimized during evolution ${ }^{68-71}$. These models incorporate mechanisms for generating variability, a component that seems to be absent in previous theories of Bayesian brains. But as evolutionary models show, it is utterly essential to account for mechanisms that create new variation, or, in the neurodynamical context, new hypotheses.

Our mechanism (Figure 1) could in principle implement, with appropriate modifications, an estimation of distribution algorithm (EDA). The population-based incremental learning (PBIL) algorithm consists of the following steps ${ }^{72}$ : (i) Generate a population from the probability vector; (ii) Evaluate and rank the fitness of each member; (iii) Update the probability vector based on the elite individual; (iv) Mutate the probability vector; (v) Repeat steps (i-iv) until a finish criterion is met. EDA can work better than copying algorithms making it an interesting line to pursue.

Future work will be to link our recurrent model with the feedforward autoencoder model of Fernando et al. ${ }^{65}$, since the latter can generate interesting genotypes (better substrates for selection) due to the emerging compressed representations of the inputs.

As two experts aptly remark: "The Bayesian brain falls short in explaining how the brain creates new knowledge" (73 p. 9). We suggest that neuronal evolutionary dynamics might serve as a remedy.

\section{Data and software availability}

Zenodo: István Zachar at doi: 10.5281/zenodo.15411374; András Szilágyi at doi: 10.5281/zenodo.80398975.

The algorithm described in the paper is also available on GitHub at https://github.com/IstvanZachar/Neurodynamics and at https:// github.com/andszilagyi/darwinianneurodyn.

\section{Author contributions}

ESz concieved the model. ASz and IZ coded the model. ASz, IZ, $\mathrm{AF}$ and HPdV contributed to the model and designed the experiments. ASz and IZ run the experiments and analyzed the data. All authors contributed to writing the manuscript.

\section{Competing interests}

No competing interests were disclosed.

\section{Grant information}

The research leading to these results has received funding from the European Union Seventh Framework Program (FP7/2007-2013) under grant agreement numbers 308943 (INSIGHT project) and 294332 (EvoEvo project). It was also supported by the project GINOP-2.3.2-15-2016-00057 (In the light of evolution).

The funders had no role in study design, data collection and analysis, decision to publish, or preparation of the manuscript.

\section{Acknowledgements}

We are thankful to Luc Steels, Chrisantha Fernando, Mauro Santos, and Thomas Filk for useful comments and discussions. We are also grateful for our reviewers, László Acsády, Stuart J. Edelstein and Karl Friston, for their invaluable comments and suggestions.

\section{Supplementary material \\ Supplementary File 1. Glossary of terms, summarizing the important evolutionary and neurobiological concepts and explaining the analogy between genetic and neuronal Darwinism.}

Click here to access the data.

Supplementary Figure S1. Analogy between genetic evolution and the attractor network-based model of Darwinian Neurodynamics. A) genetic evolution; B) attractor network-based model of Darwinian Neurodynamics. The encoded information (genotypes or attractor networks) express a trait (a phenotypic component in case of genetics; an output pattern in case of the model). Selection 
acts over these expressed traits, and the new generation's distribution of encoded information depends on this selection process. In genetic evolution, the genotype is inherited at generation $t+1$ with some mutations. In our neurodynamical model, some attractor networks (and attractors) are left unaffected, and some are altered by training them with one of the selected and mutated patterns. For more information about the analogy of Darwinian inheritance systems, see 76.

Click here to access the data.

1. Changeux JP: Neuronal man: The biology of mind. Princeton, NJ: Princeton University Press; Translated by Garey, L. 1985.

Reference Source

2. Changeux JP, Courrége $P$, Danchin A: A theory of the epigenesis of neuronal networks by selective stabilization of synapses. Proc Natl Acad Sci U S A. 1973; 70(10): 2974-2978.

PubMed Abstract | Publisher Full Text | Free Full Text

3. Edelman GM: Neural Darwinism. The theory of neuronal group selection. New York: Basic Books; 1987.

Reference Source

4. Williams RW, Rakic P: Elimination of neurons from the rhesus monkey's lateral geniculate nucleus during development. J Comp Neurol. 1988; 272(3): 424-436. PubMed Abstract | Publisher Full Text

5. O'Leary DD: Development of connectional diversity and specificity in the mammalian brain by the pruning of collateral projections. Curr Opin Neurobiol. 1992; 2(1): 70-77.

PubMed Abstract | Publisher Full Text

6. Rabinowicz T, de Courten-Myers GM, Petetot JM, et al: Human cortex development: estimates of neuronal numbers indicate major loss late during gestation. J Neuropathol Exp Neurol. 1996; 55(3): 320-328.

PubMed Abstract | Publisher Full Text

7. Miller-Fleming TW, Petersen SC, Manning L, et al.: The DEG/ENaC cation channel protein UNC-8 drives activity-dependent synapse removal in remodeling GABAergic neurons. eLife. 2016; 5: pii: e14599.

PubMed Abstract | Publisher Full Text | Free Full Text

8. Fernando C, Szathmáry E, Husbands P: Selectionist and evolutionary approaches to brain function: a critical appraisal. Front Comput Neurosci. 2012; 6: 24

PubMed Abstract | Publisher Full Text | Free Full Text

9. Quartz SR, Sejnowski TJ: The neural basis of cognitive development: a constructivist manifesto. Behav Brain Sci. 1997; 20(4): 537-556; discussion 556-96.

PubMed Abstract | Publisher Full Text

10. Bandeira F, Lent R, Herculano-Houzel S: Changing numbers of neuronal and non-neuronal cells underlie postnatal brain growth in the rat. Proc Natl Acad Sci U S A. 2009; 106(33): 14108-14113. PubMed Abstract | Publisher Full Text | Free Full Text

11. Caroni $P$, Donato $F$, Muller $D$ : Structural plasticity upon learning: regulation and functions. Nat Rev Neurosci. 2012; 13(7): 478-490. PubMed Abstract | Publisher Full Text

12. Bernardinelli $Y$, Nikonenko I, Muller D: Structural plasticity: mechanisms and contribution to developmental psychiatric disorders. Front Neuroanat. 2014; 8 : 123.

PubMed Abstract | Publisher Full Text | Free Full Text

13. Tetzlaff $\mathrm{C}$, Kolodziejski $\mathrm{C}$, Markelic I, et al.: Time scales of memory, learning, and plasticity. Biol Cybern. 2012; 106(11-12): 715-726. PubMed Abstract | Publisher Full Text

14. Maynard Smith J: The problems of biology. USA: Oxford University Press; 1986. Reference Source

15. Maynard Smith J: Genes, memes, and minds. The New York Review of Books. 1995; 42(19): 46-48.

Referen

16. Kimura M: The Neutral Theory of Molecular Evolution. Cambridge: Cambridge University Press; 1983.

Publisher Full Text

17. Eigen M: Selforganization of matter and the evolution of biological macromolecules. Naturwissenschaften. 1971; 58(10): 465-523. PubMed Abstract | Publisher Full Text

18. Adams P: Hebb and Darwin. J Theor Biol. 1998; 195(4): 419-438. PubMed Abstract | Publisher Full Text

19. Calvin WH: The brain as a Darwin Machine. Nature. 1987; 330(6143): 33-34 PubMed Abstract | Publisher Full Text

20. Calvin WH: The cerebral code: thinking a thought in the mosaics of the mind. Cambridge, MA: MIT Press. 1996 Reference Source
21. de Vladar HP, Szathmáry E: Neuronal boost to evolutionary dynamics. Interface Focus. 2015; 5(6): 20150074.

PubMed Abstract | Publisher Full Text | Free Full Text

22. Fernando $\mathrm{C}$, Goldstein R, Szathmáry $\mathrm{E}$ : The neuronal replicator hypothesis. Neural Comput. 2010; 22(11): 2809-2857. PubMed Abstract | Publisher Full Text

23. Fernando C, Karishma KK, Szathmáry E: Copying and evolution of neuronal topology. PLoS One. 2008; 3(11): e3775. PubMed Abstract | Publisher Full Text | Free Full Text

24. Fernando C, Szathmáry E: Natural selection in the brain. In: Glatzeder B, Goel V, von Müller A, editors. Towards a theory of thinking. vol. 5 of On thinking. Berlin/ Heidelberg: Springer-Verlag; 2010; 291-322. Publisher Full Text

25. Fernando C, Vasas V, Szathmáry E, et al.: Evolvable neuronal paths: a novel basis for information and search in the brain. PLOS One. 2011; 6(8): e23534. PubMed Abstract | Publisher Full Text | Free Full Text

26. Kemp C, Tenenbaum JB: The discovery of structural form. Proc Natl Acad Sci U S A. 2008; 105(31): 10687-10692.

PubMed Abstract | Publisher Full Text | Free Full Text

27. Ullman TD, Goodman ND, Tenenbaum JB: Theory learning as stochastic search in the language of thought. Cognitive Dev. The Potential Contribution of Computational Modeling to the Study of Cognitive Development: When, and for What Topics? 2012; 27(4): 455-480. Publisher Full Text

28. Börgers T, Sarin R: Learning through reinforcement and replicator dynamics. J Econ Theory. 1997; 77(1): 1-14.

Publisher Full Text

29. Niekum S, Barto AG, Spector L: Genetic programming for reward function search. IEEE Trans Auton Ment Dev. 2010; 2(2): 83-90. Publisher Full Text

30. Sutton RS, Barto AG: Introduction to reinforcement learning. 1st ed. Cambridge, MA, USA: MIT Press; 1998

Reference Source

31. Harper M: The replicator equation as an inference dynamic. ArXiv e-prints. 2009. Reference Source

32. Shalizi CR: Dynamics of Bayesian updating with dependent data and misspecified models. Electron J Statist. 2009; 3: 1039-1074. Publisher Full Text

33. Campbell JO: Universal Darwinism As a Process of Bayesian Inference. Front Syst Neurosci. 2016; 10: 49

PubMed Abstract | Publisher Full Text | Free Full Text

34. Hopfield JJ: Neural networks and physical systems with emergent collective computational abilities. Proc Natl Acad Sci U S A. 1982; 79(8): 2554-2558. PubMed Abstract | Publisher Full Text | Free Full Text

35. Rolls ET, Treves A: Neural networks and brain function. Oxford, New York: Oxford University Press; 1998.

Publisher Full Text

36. Carrillo-Reid L, Yang W, Bando Y, et al.: Imprinting and recalling cortical ensembles. Science. 2016; 353(6300): 691-694. PubMed Abstract | Publisher Full Text

37. Kilgard MP: Harnessing plasticity to understand learning and treat disease. Trends Neurosci. 2012; 35(12): 715-722. PubMed Abstract | Publisher Full Text | Free Full Text

38. Hertz J, Palmer RG, Krogh AS: Introduction to the Theory of Neural Computation. 1st ed. Perseus Publishing; 1991. Publisher Full Text

39. Storkey AJ: Efficient covariance matrix methods for Bayesian gaussian processes and Hopfield neural networks. Imperial College, Department of Electrical Engineering, Neural System Group; 1999 Reference Source

40. Watson RA, Jansen T: A building-block royal road where crossover is provably essential. In: Proceedings of the 9th Annual Conference on Genetic and Evolutionary Computation. GECCO ' 07. New York, NY, USA: ACM; 2007; 1452-1459. Publisher Full Text 
41. Maynard Smith J: The Evolution of Sex. Cambridge University Press; 1978 Reference Source

42. Maynard Smith J: The units of selection. Novartis Found Symp. 1998; 213: 203-11; discussion 211-7. PubMed Abstract

43. Sompolinsky H: Computational neuroscience: beyond the local circuit. Curr Opin Neurobiol. Theoretical and computational neuroscience. 2014; 25: xiii-xviii. PubMed Abstract | Publisher Full Text

44. Müller S, Appel B, Balke D, et al.: Thirty-five years of research into ribozymes and nucleic acid catalysis: where do we stand today? [version 1; referees: 2 approved]. F1000Res. 2016; 5: pii: F1000 Faculty Rev-1511. PubMed Abstract | Publisher Full Text | Free Full Text

45. Mountcastle VB: Modality and topographic properties of single neurons of cat's somatic sensory cortex. J Neurophysiol. 1957; 20(4): 408-434. PubMed Abstract

46. Rolls ET: Attractor networks. Wiley Interdiscip Rev Cogn Sci. 2010; 1(1): 119-134. PubMed Abstract | Publisher Full Text

47. Soto $\mathrm{D}$, Silvanto J: Reappraising the relationship between working memory and conscious awareness. Trends Cogn Sci. 2014; 18(10): 520-525. PubMed Abstract | Publisher Full Text

48. Madl T, Baars BJ, Franklin S: The timing of the cognitive cycle. PLoS One. 2011 6(4): e14803.

PubMed Abstract | Publisher Full Text | Free Full Text

49. Gustafsson B, Asztely F, Hanse E, et al:: Onset Characteristics of Long-Term Potentiation in the Guinea-Pig Hippocampal CA1 Region in Vitro. Eur J Neurosci. 1989; 1(4): 382-394. PubMed Abstract | Publisher Full Text

50. Hirsch JC, Crepel F: Use-dependent changes in synaptic efficacy in rat prefrontal neurons in vitro. J Physiol. 1990; 427(1): 31-49. PubMed Abstract | Publisher Full Text | Free Full Text

51. Fernando C, Szathmáry E: Chemical, neuronal, and linguistic replicators. In Pigliucci M, Müller G, editors. Cambridge, MA: MIT Press; 2010; 209-250. Publisher Full Text

52. Nadel L, Land C: Commentary-Reconsolidation: Memory traces revisited. Nat Rev Neurosci. 2000; 1(3): 209-212. Publisher Full Text

53. Nadel L, Moscovitch M: Memory consolidation, retrograde amnesia and the hippocampal complex. Curr Opin Neurobiol. 1997; 7(2): 217-227. PubMed Abstract | Publisher Full Text

54. Stewart TC, Choo X, Eliasmith C: Symbolic Reasoning in Spiking Neurons: A Model of the Cortex/Basal Ganglia/Thalamus Loop. In: 32nd Annual Meeting of the Cognitive Science Society; 2010. Reference Source

55. Dehaene S, Kerszberg M, Changeux JP: A neuronal model of a global workspace in effortful cognitive tasks. Proc Natl Acad Sci U S A. 1998; 95(24): 14529-14534.

PubMed Abstract | Publisher Full Text | Free Full Text

56. Shanahan M: A spiking neuron model of cortical broadcast and competition Conscious Cogn. 2008; 17(1): 288-303. PubMed Abstract | Publisher Full Text

57. Jacobs $\mathrm{C}$, Silvanto $\mathrm{J}$ : How is working memory content consciously experienced? The 'conscious copy' model of WM introspection. Neurosci Biobehav Rev. 2015; 55: 510-519.

PubMed Abstract | Publisher Full Text

58. Kumar A, Rotter S, Aertsen A: Spiking activity propagation in neuronal networks: reconciling different perspectives on neural coding. Nat Rev Neurosci. 2010; 11(9): 615-627.

PubMed Abstract | Publisher Full Text
59. Dawkins R: The evolution of evolvability. In: On growth, form and computers (eds. Kumar S, Bentley PJ): Elsevier Academic Press, London, chapter 13: 2003; 239-255.

Publisher Full Text

60. Oberauer K: Access to information in working memory: exploring the focus of attention. J Exp Psychol Learn Mem Cogn. 2002; 28(3): 411-421. PubMed Abstract | Publisher Full Text

61. Fukai T, Tanaka S: A simple neural network exhibiting selective activation of neuronal ensembles: from winner-take-all to winners-share-all. Neural Comput. 1997; 9(1): 77-97.

PubMed Abstract | Publisher Full Text

62. Morita K, Jitsev J, Morrison A: Corticostriatal circuit mechanisms of value-based action selection: Implementation of reinforcement learning algorithms and beyond. Behav Brain Res. 2016; 311: 110-121. PubMed Abstract | Publisher Full Text

63. Chklovskii DB, Mel BW, Svoboda K: Cortical rewiring and information storage. Nature. 2004; 431(7010): 782-788.

PubMed Abstract | Publisher Full Text

64. Holtmaat A, Svoboda K: Experience-dependent structural synaptic plasticity in the mammalian brain. Nat Rev Neurosci. 2009; 10(9): 647-658. PubMed Abstract | Publisher Full Text

65. Churchill AW, Sigtia S, Fernando C: Learning to generate genotypes with neural networks. Evolutionary Computation. 2015.

Reference Source

66. Maynard Smith J, Szathmáry E: The major transitions in evolution. Oxford: Freeman \& Co. 1995 Reference Source

67. Reed A, Riley J, Carraway R, et al.: Cortical map plasticity improves learning but is not necessary for improved performance. Neuron. 2011; 70(1): 121-131. PubMed Abstract | Publisher Full Text

68. Iwasa Y: Free fitness that always increases in evolution. J Theor Biol. 1988; 135 265-281.

PubMed Abstract | Publisher Full Text

69. Sella G, Hirsh AE: The application of statistical physics to evolutionary biology. Proc Natl Acad Sci U S A. 2005; 102(27): 9541-9546. PubMed Abstract | Publisher Full Text | Free Full Text

70. de Vladar HP, Barton NH: The statistical mechanics of a polygenic characte under stabilizing selection, mutation and drift. $J$ Roy Soc Interface. 2011; 8(58): 720-739.

PubMed Abstract | Publisher Full Text | Free Full Text

71. Friston K: The free-energy principle: a unified brain theory? Nat Rev Neurosc. 2010; 11(2): 127-138.

PubMed Abstract | Publisher Full Text

72. Baluja S, Caruana R: Removing the genetics from the standard genetic algorithm. Morgan Kaufmann Publishers; 1995. 38-46. Publisher Full Text

73. Friston K, Buzsáki G: The Functional Anatomy of Time: What and When in the Brain. Trends Cogn Sci. 2016; 20(7): 500-511.

PubMed Abstract | Publisher Full Text

74. Zachar I: Code for "Breeding novel solutions in the brain: A model of Darwinian neurodynamics": Publication release. 2016. Data Source

75. Szilágyi A: Code for "Breeding novel solutions in the brain: A model of Darwinian neurodynamics": Publication release. 2017. Data Source

76. Zachar I: Replicator Formalism. A general account of replication. PhD thesis. Eötvös Loránd University, Department of Plant Systematics, Ecology and Theoretical Biology. Budapest. 2011

Publisher Full Text 


\title{
Open Peer Review
}

\section{Current Peer Review Status:}

\section{Version 2}

Reviewer Report 17 July 2017

https://doi.org/10.5256/f1000research.12941.r23918

(C) 2017 Acsády L. This is an open access peer review report distributed under the terms of the Creative Commons Attribution License, which permits unrestricted use, distribution, and reproduction in any medium, provided the original work is properly cited.

\begin{abstract}
László Acsády
Laboratory of Thalamus Research, Institute of Experimental Medicine, Hungarian Academy of Sciences, Budapest, Hungary

Competing Interests: No competing interests were disclosed.

I confirm that I have read this submission and believe that I have an appropriate level of expertise to confirm that it is of an acceptable scientific standard.
\end{abstract}

Reviewer Report 03 July 2017

https://doi.org/10.5256/f1000research.12941.r23919

(C) 2017 Edelstein S. This is an open access peer review report distributed under the terms of the Creative Commons Attribution License, which permits unrestricted use, distribution, and reproduction in any medium, provided the original work is properly cited.

\section{Stuart J. Edelstein}

Ecole Normale Supérieur, Paris, France

A timely article now fully acceptable.

Competing Interests: No competing interests were disclosed.

I confirm that I have read this submission and believe that I have an appropriate level of expertise to confirm that it is of an acceptable scientific standard.

Reviewer Report 30 June 2017 


\title{
https://doi.org/10.5256/f1000research.12941.r23917
}

(C) 2017 Friston K. This is an open access peer review report distributed under the terms of the Creative Commons Attribution License, which permits unrestricted use, distribution, and reproduction in any medium, provided the original work is properly cited.

\section{Karl Friston}

Wellcome Trust Centre for Neuroimaging, University College London, London, UK

Many thanks for responding to my previous comments and congratulations on a very interesting piece.

Competing Interests: No competing interests were disclosed.

I confirm that I have read this submission and believe that I have an appropriate level of expertise to confirm that it is of an acceptable scientific standard.

\section{Version 1}

Reviewer Report 25 November 2016

https://doi.org/10.5256/f1000research.10377.r17462

(C) 2016 Acsády L. This is an open access peer review report distributed under the terms of the Creative Commons Attribution License, which permits unrestricted use, distribution, and reproduction in any medium, provided the original work is properly cited.

\author{
László Acsády \\ Laboratory of Thalamus Research, Institute of Experimental Medicine, Hungarian Academy of \\ Sciences, Budapest, Hungary
}

This is a truly enlightening and thought provoking paper which utilizes Darwinian logic to explain neuronal network dynamics. The manuscript is a nice example of how approaches in a different discipline (population genetics) may yield fresh insights into age old problems of neuroscience. I have the following notes, suggestions and questions to the authors.

Introduction:

1. One drawback of the paper is that the parallelism between genetic and neuronal Darwinism is not made clear right at the onset. We should be aware of what the authors mean by parent, offspring, multiplication, mutation, selection in case of neuronal activity right from Results: the beginning in order to follow the logic of the paper.

1. I miss the clear demonstration of the "Selection" experiments showing that it is not able to find the global optimum (e.g as an additional panel to Fig 2). I also think we need some form of quantification here, how many simulations were run, how significant the result was...etc.etc 
2. I also miss the formal demonstration of the effect of mutation rate on the speed of evolution. Since this is a crucial concept, I would dedicate a separate figure for that.

3. I would like to see, how implementing palimpsest memory affects the performance of the model and how this depends on whether the system use dense or sparse coding. Presently this is only briefly mentioned in the Method section, but since this may have important implications it may be good provide some more details. Intuitively, more sparse coding may tolerate the lack of palimpsest memory.

4. Neuronal noise considered as "mutation" in the model is enlightening. Still, I feel there are some basic differences here. During evolution genetic mutations can get stabilized when they reach the global optimum whereas neuronal noise is inherent to the system and not necessary change with evolution. Can it be demonstrated or is there any evidence that neuronal noise decreases as the system approaches the optimum state?

5. What were the connection weights of the recurrent (i.e. new input) patterns relative to the weights of the local, autoassociative connections? Can the model perform better/worse by changing the relative weights of these connections? Note that many original autoassociative models worked with a "detonator" synapse as an input and weaker local connections ${ }^{1}$.

Discussion:

1. I would not necessarily constrain the model to implicit memories. I think "implicit" here refers to the unconscious effort to recall the best target pattern not to type of memory item to be recalled. The term "implicit memory" evokes mainly procedural memories and indeed the authors place the model in the cortex-basal ganglia loop. I don't see why recall of an episodic memory trace by the CA3 recurrent network cannot follow the same evolutionary logic even though hippocampal memories are not considered as "implicit".

2. In the cortex-basal ganglia-thalamus loop, it is not really known how exactly the cortical output will affect the return signal from the thalamus but, in any case, the signal goes through significant dimension reduction ${ }^{2}$ and the final output of basal ganglia may also affect thalamic firing in different ways ${ }^{3}$ (i.e it is "mutated" a lot). The question is, how the properties of the model network changes if the final output is not directly fed back to the Minors: system but undergoes various (but consistent) signal transformation.

1. I miss the definition of "best pattern". Can this term be equated with "pattern with highest fitness"?

2. I would also support a short glossary with the neurobiological relevance of the crucial ecological concepts (fitness, generation, landscape, mutation).

3. I guess subheadings in the Results section are not appropriate. "Selection" and "Evolution" are the two main subheadings and all the others are subsections of the "Evolution" section.

\section{References}

1. Treves A, Rolls ET: Computational constraints suggest the need for two distinct input systems to the hippocampal CA3 network.Hippocampus. 1992; 2 (2): 189-99 PubMed Abstract | Publisher Full 
Text

2. Bar-Gad I, Bergman H: Stepping out of the box: information processing in the neural networks of the basal ganglia.Curr Opin Neurobiol. 2001; 11 (6): 689-95 PubMed Abstract

3. Goldberg JH, Farries MA, Fee MS: Basal ganglia output to the thalamus: still a paradox.Trends Neurosci. 2013; 36 (12): 695-705 PubMed Abstract | Publisher Full Text

Competing Interests: No competing interests were disclosed.

\section{I confirm that I have read this submission and believe that I have an appropriate level of expertise to confirm that it is of an acceptable scientific standard, however I have significant reservations, as outlined above.}

Author Response 17 Jun 2017

István Zachar, Ecology and Theoretical Biology, Institute of Biology, Eötvös University, Budapest, Hungary

We are grateful for the review of László Acsády, and his suggestions to improve our paper. We refer to the points raised by our Reviewer by a hashmark (\#). Please also see our answers to the other Reviewers for some relevant answers.

Introduction \#1. There is limited similarity only between neural Darwinism and the model and thoughts presented in this paper. In both cases "neuronal groups" are important in terms function, although the term "neuronal assembly" is more traditional and functionally relevant. Synapses are important inasmuch as they contribute to the functionality of the assembly. There is a form of reentry in both models but they play very different roles: in our case it means that the same or variant information is fed back, after evaluation, to the same population of assemblies and get stored in multiple copies. It is this multiplication component that supports "neuronal replication" of candidate solutions.

A note is in order about the search mechanism in the space of candidate solutions. In the classic Deheane-Changeux model for prefrontal cortex functionality in terms of action production and selection on the example of the Wisconsin card sorting test [1], search for new candidate solutions is random and their evaluation is strictly serial. A non-random but still serial search process, for which there is neural evidence, is chaotic itinerancy [2-4]. Except for the learning phase our attractors are locally stable, classical ones. In contrast, chaotic itinerancy rests on so-called "attractor ruins" from which there is always the possibility of dynamical escape in certain directions via transient chaotic bridges. Since these attractor ruins are deterministic entities, escape directions are not random. The pertinent question is whether these directions are random relative to those leading to better candidate solutions. One can imagine that synaptic plasticity combined with reinforcement learning might strengthen certain escape routes with experience.

An explanatory table and an accompanying figure were added to the text to guide the reader among the terms we use and to explain the relationship we imply between genetic and neuronal Darwinism. 
Results \#1. In case of Selection experiments the system can always find the global optimum, the best special pattern (it was trained to network \#20). The overlapping basins of the series of special patterns among networks guarantee that there is a trajectory from the basin of any one of the special patterns to the best special pattern. All simulations were started from noisy copies of a random input pattern similar (in Hamming-distance) to the worst special pattern. We ran 1000 independent simulations, the average number and variance of the rounds needed to converge to the optimum were $3.5 \pm 0.64$.

Results \#2. A new figure and a few sentences were added to demonstrate the effect of mutation rate on the speed of evolution.

Results \#3. Palimpsest memory is essential to our model. Because of the repeated retraining of the networks with the selected patterns, without palimpsest memory all networks - even if the patterns are sparse - sooner or later would reach catastrophic forgetting. Consequently, palimpsest memory is necessary for evolutionary processes. We used dense coding for the reason of computational time.

Results \#4. In the present state of the model, the two types of noise $\left(m_{1}\right.$ and $\left.m_{T}\right)$ are considered to be constant. Using decreasing $m_{1}$ with increasing fitness does not change the qualitative outcome of the evolutionary process (results not shown). Changes in $m_{T}$ do not affect the convergence too much, see the answer for question \#2 above.)

Results \#5. To be in line with Storkey's original model we used similar weights to the recurrent patterns and autoassociative connections. In the present state of the model, we have not tested the palimpsest behavior and the retrieval ability with other than 1:1 relative weights.

Discussion \#1, \#2. We have presented our model as potentially realisable by the cortexbasal ganglia-thalamus cortex loop. Actually, noting the massively parallel processing in the brain in general, added to the evaluative function of the mentioned loop, we would be surprised if no true evolutionary behaviour could be found during complicated problemsolving tasks. But we agree that it is not necessary to restrict the proposed cognitive architecture to this concrete loop: recall of an episodic memory trace by the CA3 recurrent network [5] can in principle follow the same evolutionary logic, even though hippocampal memories are not considered as "implicit".

A deeper question considers how "informational reentry" in the Darwinian sense can be realized by the cortex-basal ganglia-thalamus-cortex loop. In this loop it is not really known how exactly the cortical output will affect the return signal from the thalamus but, in any case, the signal goes through significant dimension reduction [6] and the final output of basal ganglia may also affect thalamic firing in different ways ([7], i.e. it is "mutated" a lot). The question is, how the properties of the model network change if the final output is not directly fed back to the system but undergoes various (but consistent) signal transformation. We think that the key to our kind of mechanism to work is exactly the consistency of signal transformation. A variant mechanism might look like this: the thalamus pointedly activates those cortical networks where the best (few) activity pattern(s) came from (as before, the number of candidate patterns "kept alive" depends on the 
stringency of selection in the striatum). These could in turn spread in the cortex through the horizontal connections among local attractors. Passing of information from attractor network to another is a common element in many relevant dynamical models $[8,9]$.

Minor points were all corrected according to Reviewer's suggestions.

References:

1 Dehaene S, Changeux JP. The Wisconsin Card Sorting Test: Theoretical Analysis and Modeling in a Neuronal Network. Cerebral Cortex. 1991;1(1):62.

http://dx.doi.org/10.1093/cercor/1.1.62.

2 Kaneko K, Tsuda I. Chaotic itinerancy. Chaos: An Interdisciplinary Journal of Nonlinear Science. 2003;13(3):926-936. http://dx.doi.org/10.1063/1.1607783. 3 van Leeuwen $C$. Chaos breeds autonomy: connectionist design between bias and babysitting. Cognitive Processing. 2008;9(2):83-92. http://dx.doi.org/10.1007/s10339-007-0193-8. 4 Tyukin I, Tyukina T, van Leeuwen C. Invariant template matching in systems with spatiotemporal coding: A matter of instability. Neural Networks. 2009;22(4):425-449. http://www.sciencedirect.com/science/article/pii/S089360800900015X.

5 Treves A, Rolls ET. Computational constraints suggest the need for two distinct input systems to the hippocampal CA3 network. Hippocampus. 1992;2(2):189-199.

http://dx.doi.org/10.1002/hipo.450020209.

6 Bar-Gad I, Bergman H. Stepping out of the box: information processing in the neural networks of the basal ganglia. Current Opinion in Neurobiology. 2001;11(6):689-695.

http://www.sciencedirect.com/science/article/pii/S0959438801002707.

7 Goldberg JH, Farries MA, Fee MS. Basal ganglia output to the thalamus: still a paradox. Trends in Neurosciences. 2013;36(12):695-705.

http://www.sciencedirect.com/science/article/pii/S0166223613001574.

8 Rolls ET, Treves A. Neural networks and brain function. Oxford, New York: Oxford

University Press; 1998. http://opac.inria.fr/record=b1094909.

9 Rolls ET. Attractor networks. Wiley Interdisciplinary Reviews: Cognitive Science.

2010;1(1):119-134. http://dx.doi.org/10.1002/wcs.1.

Competing Interests: No competing interests were disclosed.

Reviewer Report 25 October 2016

https://doi.org/10.5256/f1000research.10377.r16965

(C) 2016 Edelstein S. This is an open access peer review report distributed under the terms of the Creative Commons Attribution License, which permits unrestricted use, distribution, and reproduction in any medium, provided the original work is properly cited.

\section{Stuart J. Edelstein}

Ecole Normale Supérieur, Paris, France 
This article continues the important effort of the authors and their colleagues to advance a line of research capable of fleshing out the somewhat vague concept subsumed under the heading Neural Darwinism. While the general idea that subtle functions of the brain involve Darwinian features has persisted over the last 40 years (beginning with Changeux et al. ${ }^{1}$ ) an up-to-date synthesis has been lacking and the authors are to be congratulated for their sustained effort to achieve this goal. Part of the difficulty encountered is related to ambiguities concerning exactly what features of neo-Darwinism so successfully applied to population genetics and molecular biology, can be imported into concepts of neuroscience. In the present effort, many terms from the classical Darwinian literature are invoked, but how they are translated from population genetics to computational neuroscience is not always clear. In this respect a glossary could be very helpful, which would include the classical definition and the neural application, as applied for example to terms such as: replication, hereditary variation, breeding, fitness, mutation, deme, generation, Lamarckian, etc. In particular, the hallmark of fitness in evolution is expansion of population size, but whether and how this criterion is applicable in neuroscience should be addressed.

A second challenge is to define the level at which Darwinian principles are applied. More explicit attention could be given to dendritic spines (an obvious target for LTP, STDP), axonal pruning (see for example Kolodkin and Tessier-Lavigne ${ }^{2}$ ), or neural circuitry. Cortical columns are invoked in passing, but should be evaluated in more detail. Each of these levels is stochastic in some respects, but to what extent does the variation implicit in neural Darwinism require additional mechanisms? Concerning the results presented for the various simulations performed, several valuable points were raised in the comments by Karl Friston. In addition, it would be helpful to make clearer connections with respect to the putative neuronal structures simulated. For comparison, the recent success of deep learning algorithms should be considered, in so far as they do or do not mimic Darwinian mechanisms of the brain. Moreover, since a global optimum is set for the simulations, what criteria would establish optimality in a natural Darwinian system within the brain? Finally, what is the relationship of the high number of "generations" $(20,000$ in Figure 4) to putative neuronal processes?

Overall, describing neuronal activity using Darwinian terminology is a double-edged sword. On the one hand, a thorough application of Darwinian principles to brain science involves many oneto-one correspondences that must be clearly articulated. On the other hand, since the words are familiar, their usage carries immediate associations that can obscure understanding and become an ambiguous jargon. It would be hard to over-estimate the difficulty of finding the right balance, especially for scholars already immersed in the quest and possessing their own specific understanding of terms employed. Therefore, navigating through these troubled waters, requires extreme vigilance of language, and an addition effort by the authors in preparing their final version would be extremely helpful.

\section{References}

1. Changeux JP, Courrège $P$, Danchin $A$ : $A$ theory of the epigenesis of neuronal networks by selective stabilization of synapses.Proc Natl Acad Sci U S A. 1973; 70 (10): 2974-8 PubMed Abstract 2. Kolodkin AL, Tessier-Lavigne M: Mechanisms and molecules of neuronal wiring: a primer.Cold Spring Harb Perspect Biol. 2011; 3 (6). PubMed Abstract | Publisher Full Text

Competing Interests: No competing interests were disclosed. 


\section{I confirm that I have read this submission and believe that I have an appropriate level of expertise to confirm that it is of an acceptable scientific standard, however I have significant reservations, as outlined above.}

\section{Author Response 17 Jun 2017}

István Zachar, Ecology and Theoretical Biology, Institute of Biology, Eötvös University, Budapest, Hungary

We are extremely thankful for Stuart J. Edelstein for his comments and suggestions.

Concerning the terms we used here to relate objects and processes from neuroscience and classical Darwinism, we have included a glossary that summarizes the important evolutionary and neurobiological concepts that might be useful to clarify across disciplines. We are certain that this is not an absolute and perfect mapping of concepts, but should be enough to support our understanding of how the brain might work under Darwinian principles.

We further direct our Reviewer's attention to the answers we have provided for László Acsády on the grounding of neural Darwinism within the brain (as we understand it), especially our answers to points \#1, \#2 about the Discussion, as those also concern the questions raised here. We simply did not want to replicate our words unnecessarily.

Competing Interests: No competing interests were disclosed.

Reviewer Report 11 October 2016

https://doi.org/10.5256/f1000research.10377.r16922

(C) 2016 Friston K. This is an open access peer review report distributed under the terms of the Creative Commons Attribution License, which permits unrestricted use, distribution, and reproduction in any medium, provided the original work is properly cited.

\section{Karl Friston}

Wellcome Trust Centre for Neuroimaging, University College London, London, UK

This is a thought provoking simulation study of Darwinian neurodynamics. It uses populations of attractor networks to illustrate the distinction between purely selectionist and evolutionary optimisation. This demonstration rests upon the dynamical instability of the neuronal networks considered - and the explicit introduction of variation or mutations in graduating from a selectionist to an evolutionary scheme. The paper is rather dense and I do not pretend to follow all the subtleties and nuances; however, the basic ideas are compelling and are described with sufficient clarity and detail for the interested reader to understand. There are a few points of clarification that I think you could attend to. In addition, there are some minor grammatical improvements you could consider. 


\section{Major points}

1. I think you need to overview your simulations so that the reader knows where you are going. I would recommend something like:

"We will present a series of simulations graduating from purely selectionist schemes to evolutionary schemes in the face of a changing environment. In the first set of simulations we preclude variation in transmission over generations to examine the sufficiency of dynamical instabilities in supporting a selective process. This involves taking the outputs of one neural network and using them (after selection) to train another network. In the second set of simulations, we consider evolution proper and the transmission of patterns from generation to generation. Here, the patterns that are transmitted are subject to mild mutations (and selection) to illustrate the efficiency with which optimal (high adaptive fitness) patterns emerge."

2. I think you need to explain simply what is being optimised in your simulations. I would suggest something like:

"In what follows, we will treat a pattern of activations over binary neurons as the unit of selection. In the general case, the adaptive fitness of this pattern may be some complicated function that is contextualised by the current inputs and may or may not be a function of the history of inputs and outputs. To keep things simple, we will just consider the fitness of a pattern in terms of its hamming distance to some target pattern. This means, we are effectively using selectionist and evolutionary schemes to optimise the connections (and ensuing dynamics) to recover a target pattern."

3. When talking about the utility of dynamical instability in providing a basis for selection, you might want to refer to the work of Ivan Tyukin and colleagues ${ }^{1}, 2$. These authors have studied chaotic systems in the context optimisation - and their neuronal counterparts.

4. At the end of your discussion, I think you can usefully pursue the Bayesian brain hypothesis. I would suggest something like:

"In fact, there may be a deep connection between the selectionist dynamics illustrated in this paper and the Bayesian brain. This follows from the fact that the Bayesian brain can use Bayesian model selection to identify its most plausible hypotheses about the world. In this sense, the selective mechanisms we have demonstrated become Bayesian model selection, if we use marginal likelihood or variational free energy as adaptive fitness. See for example Sella and Hirsh, $2005^{3}$ and Friston, $2013^{4}$. Crucially, the evolutionary role of mutations and variations provides the extra ingredient required for structure learning; namely the elaboration of a model or hypothesis space."

\section{Minor points}

Page 3:

Replace "Bayesian update" with "Bayesian updates".

You might want to add a footnote about reentry and neural Darwinism when you say that "this fast scale dynamics is missing from Edelmanian neural Darwinism." I suspect that Edelman considered variation an important aspect of neural Darwinism and that this was mediated by reentrant dynamics that shows the dynamical and structural instabilities that 
you refer to.

Page 5 and throughout:

Replace "was trained only to" with "was presented only to".

Page 5:

Replace "this allows finding the global" with "this allows the system to find".

Replace "system above the", with "system above and beyond the".

I would say "... can quickly converge to the optimum - providing the output of each network is delivered to the appropriate network that successively converges on the global optimum.".

Page 6:

- Replace "at start" with "at the start".

I would remove the simulations based upon the simpler model (i.e. the thin lines in Figure 3). These simulations and their description do not add much to the text - or any insight. It would be less distracting if these simulations and their discussion were removed.

Page 8 and throughout:

Replace "provoking them" with "perturbing them".

Replace "performed of" with "performed in".

Page 8:

It would be useful to mention the (Stuart Kauffman) notion of second order selection and selection for selectability (or evolvibility). In other words, you should discuss the optimisation of the mutation rates in relation to the volatility of the environment.

Page 9:

- Replace "solves the problem" with "solving the problem".

Page 10:

Replace "networks in the given" with "networks under the given". Later replace "attractor network" with "attractors networks". Later replace "works with diluted" with "work with diluted". Finally, replace "explained in turn" with "explained next".

I hope that these comments help should any revision be required.

\section{References}

1. Tyukin I, Tyukina T, van Leeuwen C: Invariant template matching in systems with spatiotemporal coding: A matter of instability.Neural Netw. 2009; 22 (4): 425-49 PubMed Abstract | Publisher Full Text

2. van Leeuwen C: Chaos breeds autonomy: connectionist design between bias and baby-sitting. Cogn Process. 2008; 9 (2): 83-92 PubMed Abstract | Publisher Full Text

3. Sella G, Hirsh AE: The application of statistical physics to evolutionary biology.Proc Natl Acad Sci U S A. 2005; 102 (27): 9541-6 PubMed Abstract | Publisher Full Text

4. Friston K: Life as we know it.J R Soc Interface. 2013; 10 (86): 20130475 PubMed Abstract |

Publisher Full Text

Competing Interests: No competing interests were disclosed.

I confirm that I have read this submission and believe that I have an appropriate level of expertise to confirm that it is of an acceptable scientific standard. 
Author Response 17 Jun 2017

István Zachar, Ecology and Theoretical Biology, Institute of Biology, Eötvös University, Budapest, Hungary

We are thankful for Karl Friston for his invaluable comments and suggestions. Please also see our answers to the other Reviewers for some relevant answers.

About reentry and neural Darwinism: We previously discussed the limitations of Edelman's so-called "neural Darwinism" [1] in the light of standard evolutionary dynamics [2]. Here we consider two important aspects: the roles of variation and selection and reentry $[1,3]$ and how these relate to our model. In general, there are no units of evolution in the Edelman model, only units of selection. Edelman considered three subsequent phases of brain development and function (i) developmental variation and selection (supported by production and elimination of neurons and their connections), (ii) experiential selection (resting on changes in the strength of a population of synapses) and (iii) reentry (where some connections between two or more maps become stronger due to changes in synaptic weights). Note that beyond stage (i) there is no real novelty emerging. The situation is more like that in combinatorial chemistry where functional molecules are selected from a large pool of generated variants. Crucially, there is no replication with variation in this picture. Reentry has two different functions in Edelman's picture: the linking of different maps and a critical contribution to consciousness. We further direct Friston's attention to the answer we have provided for László Acsády about the realization of reentry in the cortex-basal gangliathalamus-cortex loop.

Points 1, 2. We have opted to borrow your expressions of Points 1 and 2 that perfectly summarize our original intention, and included them within the Methods section to introduce our selectionist and evolutionary experiments.

Point 3. Chaotic itinerancy is very different from our model. Our search uses the overlaps between attractors in different networks and is guided by evaluation of interim findings. Darwinian neurodynamics harnesses neuronal parallelism for search more profoundly than chaotic itinerancy.

Point 4. We have added an explanation highlighting the connection between selectionist dynamics and the Bayesian brain. We additionally cite Iwasa, who pioneered the concept as well as Barton \& de Vladar who refined it (and it would be odd not to cite some of the work of de Vladar, a co-author of this paper, who merged and developed further the ideas of Iwasa and Sella \& Hirsch).

Considering Figure 3. and the suggestion to remove the simpler model, we decided rather not to modify this section. Actually, thin lines represent the behaviour of the complex attractor network-model introduced in the paper and thick lines represent the simpler model that superficially approximate attractor dynamics without actually using networks. The whole point of this image is the comparison between our proof-of-principle attractor network model and a baseline, abstracted model. 
Certainly to Stuart Kauffman's work is an important aspect that is present in our studies. We added some explanations in this direction. However, note that the notion of evolvability is not Kauffman's (although he certainly did a good work on it and on its popularisation), but is attributed, under that name, to Dawkins and back to Riedl (1975) (but not using that term).

Most of the minor points were corrected except in a few cases where they went explicitly against our intended meaning. For example we rather stick with the word "provocation", as "perturbation" has a different meaning to what we intended here.

References:

1 Edelman GM. Neural Darwinism. The theory of neuronal group selection. New York: Basic Books; 1987.

2 Fernando C, Szathmáry E, Husbands P. Selectionist and evolutionary approaches to brain function: a critical appraisal. Frontiers in Computational Neuroscience. 2012;6(24).

http://dx.doi.org/10.3389/fncom.2012.00024.

3 Edelman GM. Neural Darwinism: Selection and reentrant signaling in higher brain function. Neuron. 1993;10(2):115-125.

http://www.sciencedirect.com/science/article/pii/089662739390304A.

Competing Interests: No competing interests were disclosed.

The benefits of publishing with F1000Research:

- Your article is published within days, with no editorial bias

- You can publish traditional articles, null/negative results, case reports, data notes and more

- The peer review process is transparent and collaborative

- Your article is indexed in PubMed after passing peer review

- Dedicated customer support at every stage

For pre-submission enquiries, contact research@f1000.com 